\title{
The Role of PSD-95 and Cypin in Morphological Changes in Dendrites Following Sublethal NMDA Exposure
}

\author{
Chia-Yi Tseng ${ }^{1,2}$ and Bonnie L. Firestein ${ }^{1}$ \\ ${ }^{1}$ Department of Cell Biology and Neuroscience, ${ }^{2}$ The Graduate Program in Neuroscience, Rutgers, The State University of New Jersey, Piscataway, New \\ Jersey 08854-8082
}

Focal swelling or varicosity formation in dendrites and loss of dendritic spines are the earliest indications of glutamate-induced excitotoxicity. Although it is known that microtubule dynamics play a role in varicosity formation, very little is known about the proteins that directly impact microtubules during focal swelling and dendritic spine loss. Our laboratory has recently reported that the postsynaptic protein PSD-95 and its cytosolic interactor (cypin) regulate the patterning of dendrites in hippocampal neurons. Cypin promotes microtubule assembly, and PSD-95 disrupts microtubule organization. Thus, we hypothesized that cypin and PSD-95 may play a role in altering dendrite morphology and spine number in response to sublethal NMDA-induced excitotoxicity. Using an in vitro model of glutamateinduced toxicity in rat hippocampal cultures, we found that cypin overexpression or PSD-95 knockdown increases the percentage of neurons with varicosities and the number of varicosities along dendrites, decreases the size of varicosities after sublethal NMDA exposure, and protects neurons from NMDA-induced death. In contrast, cypin knockdown or PSD-95 overexpression results in opposite effects. We further show that cypin regulates the density of spines/filopodia: cypin overexpression decreases the number of protrusions per micrometer of dendrite while cypin knockdown results in an opposite effect. Cypin overexpression and PSD-95 knockdown attenuate NMDA-promoted decreases in protrusion density. Thus, we have identified a novel pathway by which the microtubule cytoskeleton is regulated during sublethal changes to dendrites.

\section{Introduction}

Early results of glutamate-induced neuronal injury observed in vivo and in vitro include focal swelling/varicosities in dendrites, spine loss, and cytoskeletal degradation (Hasbani et al., 1998, 2001; Ikegaya et al., 2001; Monnerie et al., 2003; Greenwood et al., 2007). Disintegrated and fragmented microtubules are found in swollen dendrites, inside of varicosities (Yamamoto et al., 1986; Tomimoto and Yanagihara, 1992; Greenwood et al., 2007). It is thought that microtubule integrity relates to varicosity formation and dendrite recovery (Faddis et al., 1997). Indeed, Taxol, the microtubule stabilizer, attenuates glutamate-induced varicosity formation (Emery and Lucas, 1995; Furukawa and Mattson, 1995; Park et al., 1996). In addition, spine retraction and reemergence are associated with modification of cytoskeletal organization. Stabilization of actin filaments reduces dendritic spine loss after ischemia (Gisselsson et al., 2005; Meller et al., 2008). However, actin polymerization does not contribute to spine recovery (Hasbani et al., 2001). Thus, the precise molecular mechanisms

\footnotetext{
Received May 16, 2011; revised Aug. 26, 2011; accepted Sept. 5, 2011.

Author contributions: C.-Y.T. and B.L.F. designed research;C.-Y.T. performed research; C.-Y.T. and B.L.F. analyzed data; C.-Y.T. and B.L.F. wrote the paper.

This work was supported in part by a Busch Biomedical Grant, National Science Foundation Grants IBN-0919747 and IBN-0548543, March of Dimes Foundation Grants 1-FY04-107 and 1-FY08-464, and a Grant-In-Aid from the American Heart Association (to B.L.F). We thank Martin Schwander and members of the Firestein Laboratory for their comments on this manuscript.

Correspondence should be addressed to Dr. Bonnie L. Firestein, Department of Cell Biology and Neuroscience, Rutgers, The State University of New Jersey, Nelson Biological Laboratories, 604 Allison Road, Piscataway, NJ 088548082. E-mail: Firestein@biology.rutgers.edu.

DOI:10.1523/JNEUROSCI.2442-11.2011

Copyright $\odot 2011$ the authors $\quad 0270-6474 / 11 / 3115468-13 \$ 15.00 / 0$
}

that regulate dendrite integrity and spine morphogenesis in ischemia remain largely unknown.

Although dendritic varicosity formation is seen in a number of conditions, including disease, traumatic brain injury, and ischemic stroke, a number of recent studies have focused on the timing and role of glutamate receptors in mediating these changes in the intact animal during ischemic stroke (Brown et al., 2007; Murphy et al., 2008). While these studies are of high importance, it is difficult to assess the roles of intracellular proteins in mediating dendritic changes in vivo, since viral infection or transfection to alter specific protein levels in neurons undergoing these changes is technically challenging.

We have chosen to use an in vitro model, primary cultures of hippocampal neurons exposed to sublethal concentrations of NMDA, to study the roles of two proteins, postsynaptic density-95 protein (PSD-95) and its cytosolic interactor (cypin), known to regulate cytoskeletal dynamics in dendrites. We previously reported that PSD-95 decreases dendrite branching by disrupting microtubule organization (Charych et al., 2006; Sweet et al., 2011a,b). Moreover, PSD-95 is in a complex with F-actin, and PSD-95 overexpression enhances dendritic spine formation and stabilization (Okabe et al., 1999; El-Husseini et al., 2000; Horne and Dell'Acqua, 2007). Cypin increases dendrite branching by promoting microtubule polymerization and negatively regulating PSD-95 clustering (Firestein et al., 1999; Akum et al., 2004; Charych et al., 2006). Accordingly, cypin and PSD-95 may be involved in the machinery that maintains dendrite integrity and promotes spine retraction and reemergence in response to sublethal glutamate exposure. 
A

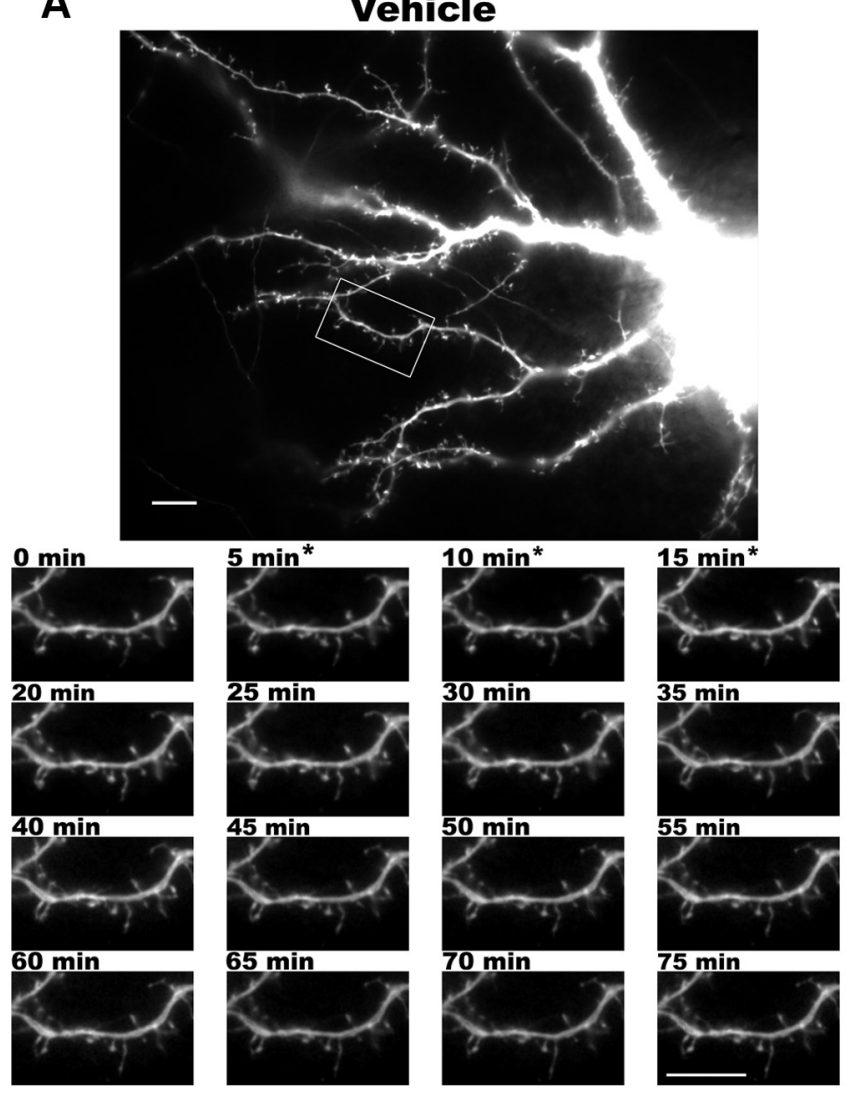

B
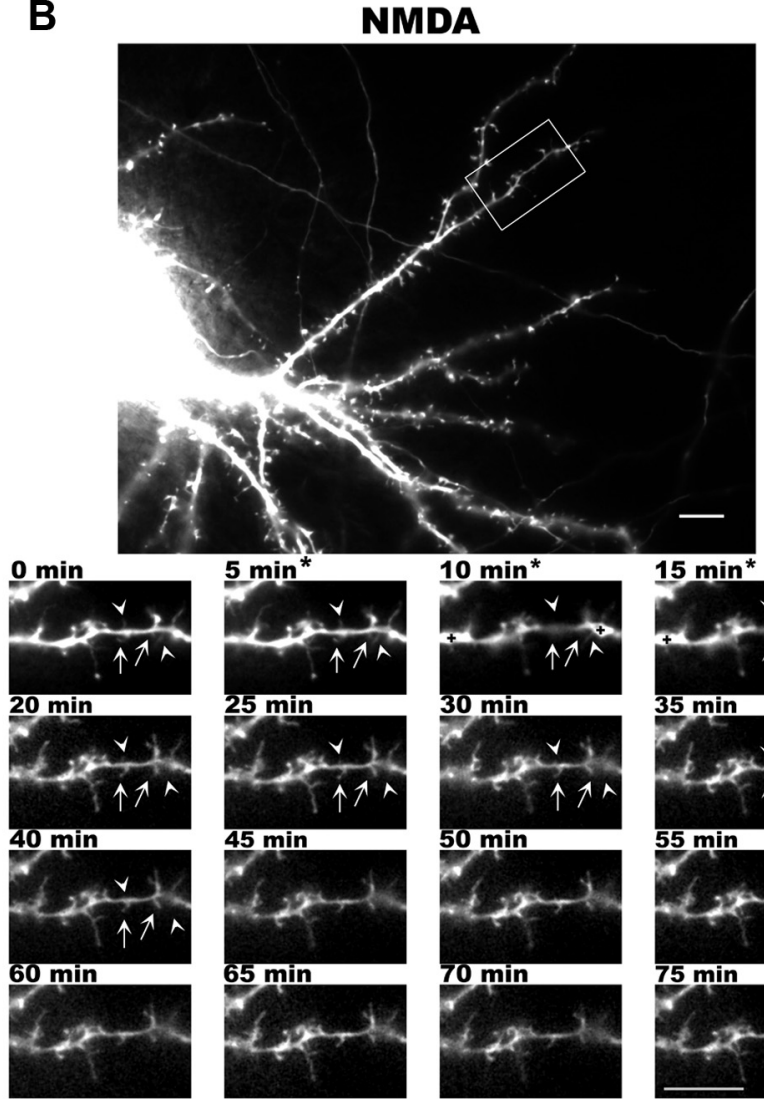

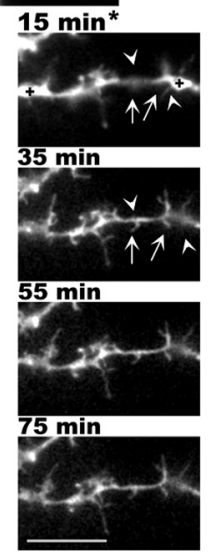

Figure 1. Changes in dendrite morphology following sublethal excitotoxic exposure. Primary hippocampal neurons were transfected with cDNAs encoding GFP on DIV 14. Live cell time-lapse imaging was performed at DIV 20, and frames were collected at 5 min intervals for 75 min. Neurons were observed for 5 min before $30 \mu \mathrm{m} \mathrm{NMDA}$ or vehicle treatment, 10 min during $30 \mu \mathrm{m}$ NMDA application (asterisk) or vehicle treatment, and $60 \mathrm{~min}$ after returning to recovery medium. Representative live cell time-lapse imaging is shown. $A$, Dendrite and protrusion morphology remains stable in the vehicle-treated condition. $\boldsymbol{B}$, Varicosities form within minutes after application of $30 \mu \mathrm{M} \mathrm{NMDA}$ (plus symbol) and a number of them persist after recovery for 60 min. A number of protrusions retract during NMDA treatment. Some of the retracted protrusions reemerge when the neurons are returned to recovery medium (arrow) while some do not (arrowhead). Scale bars: $10 \mu \mathrm{m}$.

To elucidate the roles of PSD-95 and cypin in mediating changes in dendrite morphology, we applied a sublethal dose of NMDA to primary hippocampal neurons. We find that microtubules are disrupted in response to this treatment. Furthermore, overexpression of cypin and knockdown of PSD-95 result in decreased varicosity size but increased percentage of neurons with varicosities (beaded neurons) and number of varicosities per micrometer of dendrite after sublethal NMDA treatment. Furthermore, neuroprotection from NMDA-induced excitotoxicity is seen under these conditions. We see opposite effects when cypin is overexpressed and PSD-95 is knocked down. Moreover, overexpression of cypin and knockdown of PSD-95 result in a reduction in the loss of dendritic protrusions in response to NMDA treatment. Our results suggest that PSD-95 and cypin are part of a signaling pathway that regulates changes in the morphology of dendrites and dendritic protrusions after sublethal NMDA exposure.

\section{Materials and Methods}

All animal studies were approved by the Rutgers University Animal Care and Facilities Committee.

Antibodies. Rabbit polyclonal anti-ribosomal protein L9 (Rpl9) was purchased from Sigma. Mouse monoclonal anti-PSD-95 was purchased from ThermoFisher Scientific. Rabbit polyclonal anti-microtubule-associated protein 2 (MAP2) and mouse monoclonal anti-glyceraldehyde 3-phophate dehydrogenase (GAPDH) were purchased from Millipore. Chicken poly- clonal anti-GFP was purchased from Rockland Immunochemicals. Mouse monoclonal anti-neuronal class III $\beta$-tubulin (Tuj1) was from Covance. Mouse monoclonal anti-lactate dehydrogenase antibody and rabbit polyclonal anti-synapsin I were from Abcam. Rabbit polyclonal anti-PSD-95 and rabbit polyclonal anti-cypin were characterized previously (Firestein et al., 1999). Cyanine (Cy)2-, Cy3-, and Cy5-conjugated secondary antibodies were from Jackson ImmunoResearch. HRP-conjugated anti-rabbit and anti-mouse antibodies were purchased from VWR International.

Neuronal culture and transfection. Neuronal cultures were prepared from hippocampi of rat embryos of both sexes at $18 \mathrm{~d}$ of gestation as described previously (Firestein et al., 1999). Hippocampi were dissociated, and cells were plated on glass coverslips (12 $\mathrm{mm}$ in diameter), 35 $\mathrm{mm}$ glass bottom Petri dishes, or $35 \mathrm{~mm}$ Petri dishes coated with polyD-lysine at a density of $600-800$ cells $/ \mathrm{mm}^{2}$. Neurons were cultured in Neurobasal media supplemented with B27, penicillin, streptomycin, and GlutaMax (Invitrogen). For plasmid transfection, neurons were grown for $14 \mathrm{~d}$ in culture (DIV 14) and transfected with the appropriate constructs using Effectene (Qiagen) following the manufacturer's instructions, except for cell viability studies where neurons were transfected using the calcium phosphate method (Xia et al., 1996). The sequence for the PSD-95 shRNA used for our studies is $5^{\prime}$-GCCTTCGACAGAGCCA CGA-3' and 5'-GCCTTCGATCGTGCCACGA-3' for the rescue mutant. We previously published the sequence for the cypin shRNA and rescue plasmid (Chen and Firestein, 2007). All overexpression plasmids have been previously published (Akum et al., 2004; Charych et al., 2006). Neurons were allowed to express the exogenous protein for $6 \mathrm{~d}$ and were then subjected to NMDA treatment as described below. 
NMDA treatment, immunocytochemistry, and imaging. Treatment with a sublethal dose of NMDA (Sigma) was performed as previously described (Hasbani et al., 1998) with some modification. All steps were performed at $37^{\circ} \mathrm{C}$. NMDA was diluted in HEPES-buffered balanced salt solution (HBBSS; $200 \mathrm{mg} / \mathrm{l}$ $\mathrm{CaCl}_{2}, 400 \mathrm{mg} / \mathrm{l} \mathrm{KCl}, 77.3 \mathrm{mg} / \mathrm{l} \mathrm{MgCl}, 3 \mathrm{~g} / \mathrm{l}$ $\mathrm{NaCl}, 2.2 \mathrm{~g} / \mathrm{l} \mathrm{NaHCO} 3,125 \mathrm{mg} / \mathrm{l} \mathrm{NaH} \mathrm{NO}_{4}$ $\mathrm{H}_{2} \mathrm{O}, 4.5 \mathrm{~g} / \mathrm{l}$ D-glucose, $2.6 \mathrm{~g} / \mathrm{l} \mathrm{HEPES}, \mathrm{pH}$ 7.4). Briefly, on DIV 20, neurons expressing the appropriate constructs were exposed to $30 \mu \mathrm{M}$ NMDA for $10 \mathrm{~min}$. In parallel, sister cultures were treated only with HBBSS buffer to serve as controls. At the end of NMDA application, recovery groups were returned to HBBSS buffer containing $10 \mu \mathrm{M}$ MK-801 (Sigma).

For fixed cell time-lapse imaging, exposures or recoveries were terminated by fixation in $4 \%$ paraformaldehyde in PBS for 10 min at the appropriate time points. Neurons were visualized by immunofluorescence under a $60 \times$ oilimmersion objective on an Olympus IX50 microscope with a Cooke Sensicam CCD-cooled camera, fluorescence, imaging system, and Image Pro software. The experimenter was blinded to the condition when taking images and analyzing dendrite and spine morphology. For cell viability experiments, fixed neurons were stained with rabbit anti-MAP2 (1:500) and chicken anti-GFP (1:500) followed by secondary antibodies conjugated to Cy2 or Cy5. Nuclei were stained using DAPI (Sigma) to determine cell survival. Stained neurons were visualized by immunofluorescence under a $10 \times$ objective. Thirty different objective views were randomly selected from three independent experiments. Only GFP-positive neurons with clear neuronal morphology were examined. The experimenter was blinded to the condition when taking images and counting. Neurons with negative MAP2 immunostaining and condensed DAPI staining were considered dead.

For live cell time-lapse imaging, culture medium was replaced with HBBSS buffer for $30 \mathrm{~min}$ before imaging, and neurons were placed onto a heated stage. Images were acquired using the same equipment as described above at $5 \mathrm{~min}$ intervals for up to $75 \mathrm{~min}$. One picture was captured before adding NMDA, followed by three pictures during NMDA application, and finally, 13 pictures during the recovery stage. To modify microtubule organization, either $10 \mu \mathrm{g} / \mathrm{ml}$ Taxol (Sigma) or 10 $\mu \mathrm{g} / \mathrm{ml}$ nocodazole (Sigma) was applied $30 \mathrm{~min}$ before NMDA treatment until the end of recording. Control sister cultures were exposed to vehicle conditions. For examination of presynaptic contacts for protrusions, neurons were fixed with $4 \%$ paraformaldehyde in PBS for 10 min immediately after the end of recovery. Neurons were stained with chicken anti-GFP (1:500) and rabbit anti-synapsin I (1:500) followed by secondary antibodies conjugated to Cy2 and Cy5. Dendrites at least $10 \mu \mathrm{m}$ away from the soma were randomly chosen, and the percentage of protrusions that appose immunostained clusters of the presynaptic marker synapsin I was determined. In addition to fixed cells, at least three live cells, imaged over time, were examined.

For endogenous PSD-95 cluster immunostaining, neurons expressing the appropriate constructs were fixed on DIV 20. Neurons were stained with mouse anti-PSD-95 (1:500), anti-synapsin I (1:500), and chicken anti-GFP (1:500) followed by secondary antibodies conjugated with Cy2, Cy3, and Cy5. Dendrites at least $10 \mu \mathrm{m}$ away from the soma were randomly chosen, and the percentage of PSD-95 clusters that appose synapsin I immunostaining versus total number of clusters was counted.

Statistical significance $(p<0.05)$ was determined using ANOVA followed by the appropriate post hoc test using GraphPad InStat or GraphPad Prism software. $10 \mu \mathrm{m}$.
0 min
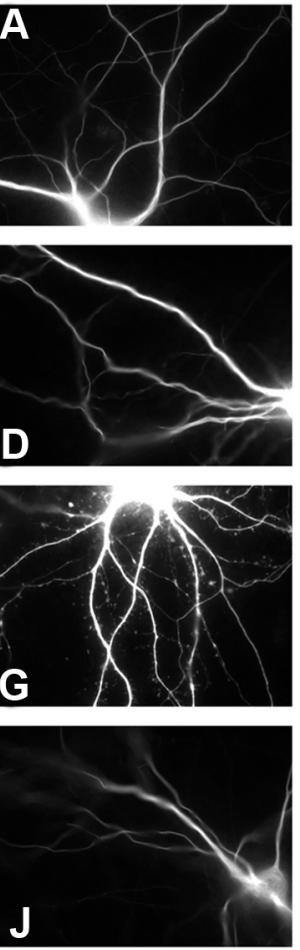

$15 \mathrm{~min}$
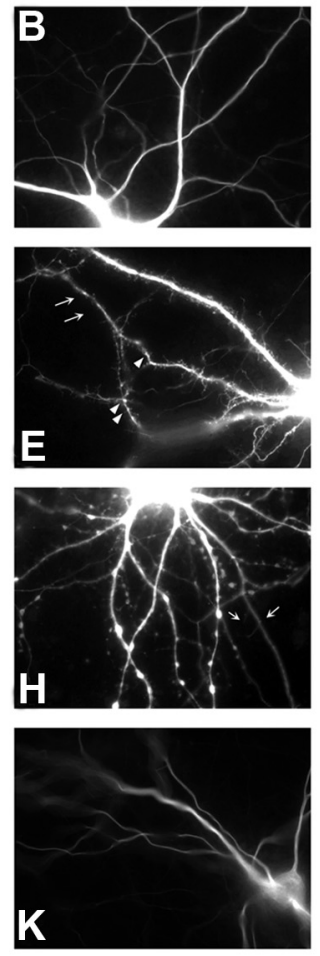

$75 \mathrm{~min}$
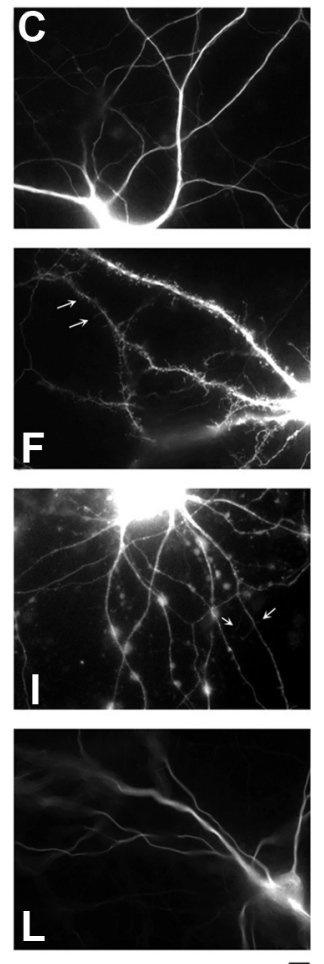

Figure 2. The organization of microtubules is disturbed after exposure to sublethal NMDA. Primary hippocampal neurons were transfected with CDNAs encoding $\alpha$-tubulin-GFP on DIV 14. On DIV 20, neurons were treated with vehicle or $30 \mu \mathrm{M}$ NMDA. $A-L$,

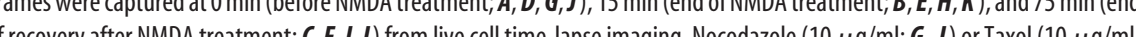
$J$ ) was applied to the cultures $30 \mathrm{~min}$ before NMDA treatment until the end of recording. Arrowheads in $\boldsymbol{E}$ indicate regions of swelling. Arrows in $\boldsymbol{E}$ and $\boldsymbol{F}$ or $\boldsymbol{H}$ and $\boldsymbol{I}$ indicate that protrusions that contain $\alpha$-tubulin-GFP that are longer after recovery. Scale bar, lyzed using NIH ImageJ software. Quantitative analyses of dendrite and protrusion morphology are from fixed cell time-lapse imaging. A dendritic swelling is considered a varicosity when it is twice the width of the dendritic shaft. Neurons were considered beaded if they had three varicosities along the same dendrite. Thirty neurons from three independent experiments were counted at each time point of each group. Data were expressed as percentage beaded neurons (normalized to total neurons counted). In a beaded neuron, three dendrites at least $10 \mu \mathrm{m}$ away from the soma were randomly chosen, and the number of beaded structures along the same $10 \mu \mathrm{m}$ length of dendrite was counted. The data were expressed as the number of varicosities per micrometer of dendrite. In addition, the area of each varicosity on the same dendrite was examined, and the result was represented as varicosity size $\left(\mu \mathrm{m}^{2}\right)$.

Both filopodia and spines were counted as protrusions in order not to rule out any alterations in spine morphology due to NMDA-induced excitotoxicity (Hasbani et al., 2001). Two dendrites per neuron were randomly chosen, and the number of protrusions from a particular length of dendrite $(10 \mu \mathrm{m})$ was counted. The result was expressed as number of protrusions per $\mu \mathrm{m}$ of dendrite. Statistical differences $(p<$ 0.05 ) were determined using ANOVA followed by the appropriate post hoc test using GraphPad InStat or GraphPad Prism software.

Western blotting. Hippocampal cultures (DIV 20) were scraped into $150 \mu$ of TEE (20 mm TrisHCl pH 7.4, 1 mm EDTA, 1 mm EGTA) with $1 \%$ Triton X-100 at the appropriate time points after NMDA application. Neurons were homogenized and lysed by passage through a 25.5 gauge needle 20 times. The homogenates were spun at $1000 \times g$ for $10 \mathrm{~min}$. For analysis of proteins released into the medium, HBBSS buffer (medium) was collected at the appropriate time points after NMDA application. Amicon Ultra-4 Centrifugal Filter Unit with Ultracel-10 membrane (Millipore) was used to concentrate the medium 20-fold. Bradford assays 
EXTRACT

A

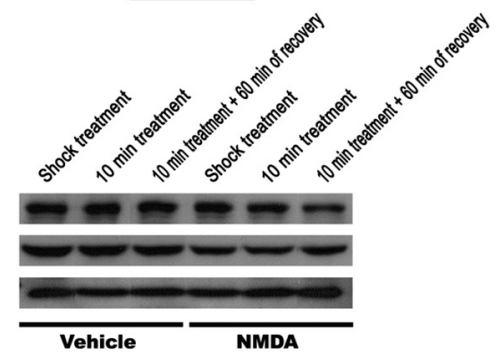

B
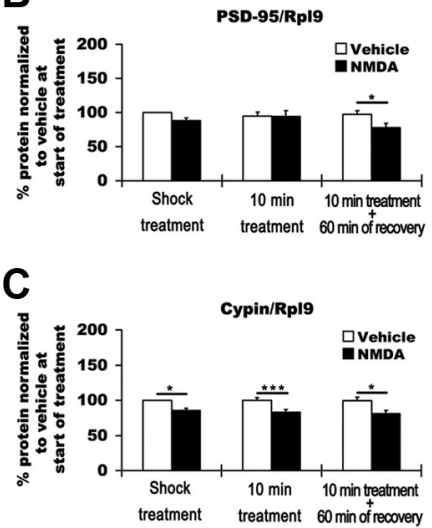

$\underline{\text { MEDIA }}$
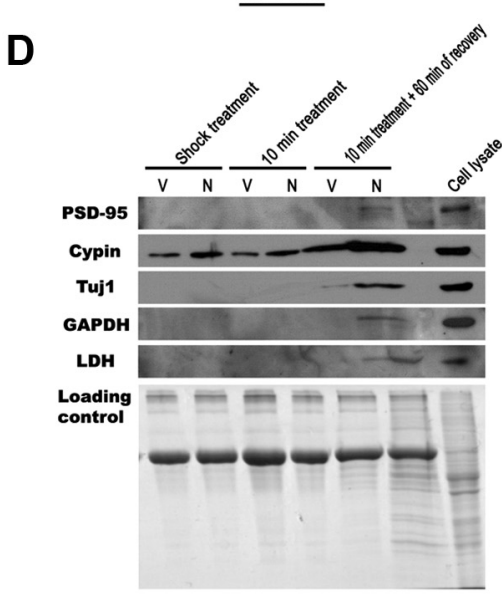

E

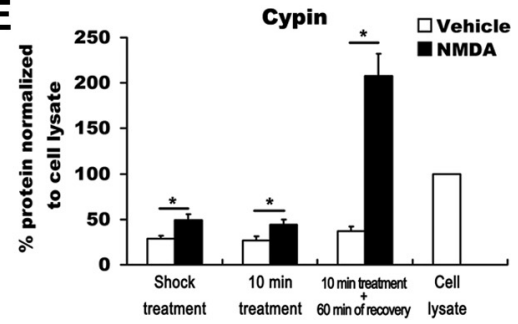

Figure 3. Effects of sublethal NMDA exposure on cypin protein levels. At DIV 20, neurons were exposed to vehicle (V) or $30 \mu \mathrm{M}$ $\operatorname{NMDA}(\mathrm{N})$ for $10 \mathrm{~min}$ followed by 60 min in recovery medium. $\boldsymbol{A}-\boldsymbol{E}$, Cell lysates (Extract; $\boldsymbol{A}-\boldsymbol{C}$ ) and HBBSS buffer (Media; $\boldsymbol{D}, \boldsymbol{E}$ ) were collected at indicated periods. The homogenized samples and concentrated medium were subjected to SDS-PAGE and Western blotting using antibodies to PSD-95, cypin, Rpl9, Tuj1, LDH, and GAPDH. To normalize for total protein from media loaded in media, SDS-polyacrylamide gels were stained with Coomassie Blue. For analysis of proteins in extract, PSD-95 and cypin band intensities were normalized to Rpl9 band intensity. $\boldsymbol{A}$, Representative Western blot of extract from five independent experiments is shown. $\boldsymbol{B}$, Quantitative analysis of PSD-95/Rpl9 band intensity shows a significant decrease in PSD-95 protein after recovery. C, Quantitative analysis of cypin/Rpl9 band intensity shows lower cypin protein levels at all time points with NMDA treatment. $\boldsymbol{D}$, Representative Western blot of proteins in medium from four independent experiments is shown. $E$, Quantitative analysis of cypin band intensity shows an increase in cypin released into the medium after NMDA treatment. ${ }^{*} p<0.05,{ }^{* *} p<0.01,{ }^{* * *} p<0.001$ by Friedman test (repeated-measures nonparametric ANOVA) followed by Dunn's multiple-comparisons test.

were used to determine the protein concentrations of the extracts. Proteins were resolved on a $10 \%$ SDS-polyacrylamide gel and transferred to a PVDF membrane. The blot was probed with the indicated antibodies. When extract was being analyzed, expression of Rpl9 was used to normalize the amount of total protein loaded into each well since it has been shown that Rpl9 does not change during ischemic conditions (Kobayashi et al., 2004). For analysis of proteins released into the medium, the SDS-polyacrylamide gel was stained using Coomassie Blue to confirm equal amounts of total protein loaded into each well. Experiments were repeated at least three times. Quantification of blots was performed as previously described with modification (Chen and Firestein, 2007). Briefly, the blots were scanned, and the total integrated density of bands was analyzed using ImageJ. An area with the same size of the selected region close to the bands was used to account for background intensity. The difference between integrated density of the background and the band was determined to be the absolute intensity of the band.

\section{Results}

The morphologies of dendrites and dendritic protrusions change in response to sublethal NMDA-induced excitotoxicity

We have chosen to use an in vitro model of glutamate-induced excitotoxicity since viral infection or transfection to alter specific protein levels in vivo is technically challenging. Previous reports show that glutamate-induced morphological changes in dendrites can be mimicked in culture by activating NMDA receptors (Hasbani et al., 1998; Hou et al., 2002; Monnerie et al., 2003; Zhang et al., 2007;
Wahl et al., 2009). Moreover, application of sublethal NMDA receptor agonists successfully reproduces glutamateinduced excitotoxicity, which results in varicosity formation and loss of dendritic spines within minutes. In addition, dendrite and spine morphology are restored within $2 \mathrm{~h}$ after washout of the glutamate receptor activator (Park et al., 1996; Faddis et al., 1997; Hasbani et al., 1998, 2001; Wilson and Keith, 1998; Inoue and Okada, 2007).

Here, we first characterized sublethal NMDA-induced morphological changes in dendrites in primary hippocampal neurons since most studies have focused on cortical neurons (Hasbani et al., 1998, 2001; Inoue and Okada, 2007). We determined that treatment of our cultures with $30 \mu \mathrm{M}$ NMDA for $10 \mathrm{~min}$ resulted in $<50 \%$ neuronal death (data not shown). Using live cell time-lapse imaging, we found that varicosities formed along dendrites within minutes of NMDA exposure and that a number of protrusions retracted immediately after NMDA application (Fig. 1B). Although the GFP fluorescence appeared dimmer within 60 min after returning the cultures to recovery medium, possibly due to breakdown of neuronal membranes, a number of the NMDAinduced varicosities persisted, and a subset of protrusions reemerged. In addition, dendrite morphology remained unchanged and dendritic protrusions were stable in the vehicle condition (Fig. 1A). Therefore, our in vitro sublethal dose model successfully mimics glutamate-induced acute morphological changes in dendrites seen in cortical neurons in previous studies.

\section{Organization of microtubules is disrupted after NMDA exposure}

Microtubules have been reported to play a role in NMDAinduced alterations in dendrite morphology (Park et al., 1996; Faddis et al., 1997; Hasbani et al., 1998). Disintegrated microtubules have been found in swollen dendrites (Yamamoto et al., 1986; Tomimoto and Yanagihara, 1992), and disruptions of microtubules appear inside of varicosities (Greenwood et al., 2007). Accordingly, we asked whether changes in microtubule organization can be observed in our in vitro model of sublethal NMDAinduced excitotoxicity. To address the question, neurons were transfected with cDNA encoding GFP-tagged $\alpha$-tubulin at DIV 14. Sublethal NMDA treatment and live cell time-lapse imaging was performed at DIV 20. We found that filamentous $\alpha$-tubulinGFP along the dendrites was discontinuous (Fig. 2E), and varicosity-like swelling structures (Fig. $2 E$, arrowhead) formed along the dendrites at the end of NMDA treatment. After recovery, swelling was ameliorated (Fig. $2 F$ ). Moreover, $\alpha$-tubulinGFP entered dendritic protrusions after NMDA application (Fig. $2 E, F)$. A number of $\alpha$-tubulin-GFP-positive protrusions were longer after recovery (Fig. 2E, F, arrow). In addition, filamentous dis- 
tribution of $\alpha$-tubulin-GFP along the dendrite remained unchanged during vehicle treatment (Fig. 2A-C).

We then asked whether pharmacological manipulations of the cytoskeleton could alter dendritic swelling after sublethal NMDA application in our in vitro model. Taxol, a microtubule stabilizer, has been shown to reduce dendritic swelling in response to sublethal stimulation of the NMDA receptor (Emery and Lucas, 1995; Furukawa and Mattson, 1995; Park et al., 1996). Taxol was applied to neurons 30 min before NMDA treatment until the end of recording. We found that filamentous $\alpha$-tubulin-GFP along dendrites remained intact and stable during and after NMDA treatment (Fig. $2 J-L$ ). In addition, we treated neurons with nocodazole, a microtubule-depolymerizing agent. We observed that microtubules were disrupted before NMDA treatment (Fig. $2 G)$. As expected, a more severe disorganization of microtubules was observed after NMDA receptor activation, which persisted throughout the recovery period (Fig. $2 H, I$ ). A number of $\alpha$-tubulin-GFPpositive protrusions were longer after recovery (Fig. $2 \mathrm{H}, \mathrm{I}$, arrow). Moreover, $\alpha$-tubulin-GFP fluorescence was dimmer in nocodazole-treated neurons than in control neurons. Together, our data suggest that the disruption of microtubule organization plays a role in varicosity formation in neurons exposed to sublethal concentrations of NMDA. Our data also show that tubulin enters into dendritic protrusions after NMDA application, elucidating the potential role of microtubules in NMDA-mediated changes in dendrite morphology.

\section{PSD-95 and cypin protein decrease after exposure to a sublethal dose of NMDA}

Because PSD-95 and cypin play a role in the modification of microtubule dynamics (Akum et al., 2004; Charych et al., 2006; Chen and Firestein, 2007; Sweet et al., 2011a,b), we next asked whether protein levels of PSD-95 and cypin may change in parallel with the alteration of microtubule organization after sublethal NMDA application. We performed Western blot analysis from cellular extracts to determine protein expression of PSD-95 and cypin with shock NMDA treatment, after $10 \mathrm{~min}$ of NMDA treatment, and after $10 \mathrm{~min}$ of NMDA treatment followed by 60 min recovery. Control neurons were exposed to vehicle treatment. There is no significant difference in the levels of Rpl9 protein, which should not change in response to treatment (Kobayashi et al., 2004), between control and NMDA-treated groups (data not shown). We found that PSD-95 protein significantly decreases after 10 min treatment followed by 60 min recovery in the NMDA-treated group (Fig.

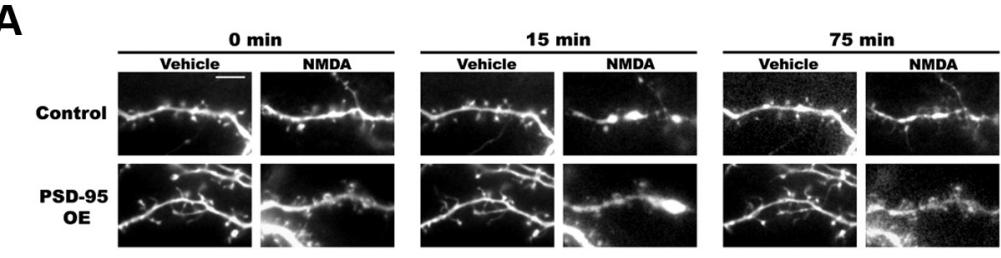

B

D

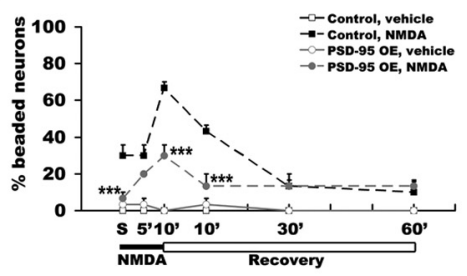

C

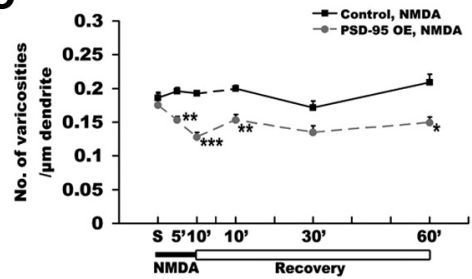

E

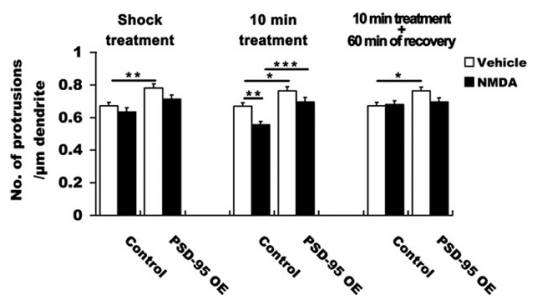

Figure 4. The effects of overexpression of PSD-95 on NMDA-induced changes in dendrite morphology. Primary hippocampal neurons were cotransfected with cDNAs encoding GFP and RFP (control) or PSD-95-RFP [PSD-95 overexpression (PSD-95 OE)] at DIV 14. At DIV 20, neurons were exposed to vehicle or $30 \mu \mathrm{M} \mathrm{NMDA}$ for $10 \mathrm{~min}$ followed by 60 min in recovery medium. $\boldsymbol{A}$, Representative live cell time-lapse imaging was captured at $0 \mathrm{~min}$ (before treatment), $15 \mathrm{~min}$ (end of treatment), and $75 \mathrm{~min}$ (end of recovery). Scale bar, $5 \mu \mathrm{m}$. $\boldsymbol{B}-\boldsymbol{E}$, Quantitative analyses of time-lapse experiments from fixed cells imaging of sister neuronal cultures from three independent experiments. $\boldsymbol{B}$, Varicosity size. Number of varicosities analyzed at each time point of each group in NMDA treatment, $n=178$ after NMDA treatment followed by 10 min recovery, $n=47$ after NMDA treatment followed by 30 min recovery, and $n=43$ after NMDA treatment followed by 60 min recovery; in PSD-95 OE, $n=24$ at $S$ with NMDA treatment, recovery, $n=37$ after NMDA treatment followed by 30 min recovery, and $n=41$ at after NMDA treatment followed by 10 min recovery. ${ }^{*} p<0.05,{ }^{* *} p<0.001$ by Kruskal-Wallis test followed by Dunn's multiple-comparisons test compared with control NMDA. C, Number of varicosities per micrometer of dendrite. Number of beaded dendrites analyzed at each time point of each group is as follows: for control, $n=27$ at $S$ with NMDA treatment, $n=27$ at 5 min with NMDA treatment, $n=60$ at 10 min with NMDA treatment, $n=39$ after NMDA treatment followed by 10 min recovery, $n=12$ after NMDA treatment followed by 30 min recovery, and $n=9$ after NMDA treatment followed by 60 min recovery; for PSD-95 0E, $n=6$ at $S$ with NMDA treatment, $n=18$ at 5 min with NMDA treatment, $n=27$ at 10 min with NMDA treatment, $n=12$ after NMDA treatment followed by 10 min recovery. ${ }^{* *} p<0.01,{ }^{* * *} p<0.001$ by Kruskal-Wallis test followed by Dunn's multiple-comparisons test compared with control NMDA. $\boldsymbol{D}$, Percentage beaded neurons. Thirty neurons were analyzed at each time point of each group. ${ }^{* * *} p<0.001$ by two-way Donferroni multiple-comparisons test. The plot only shows the comparison between control NMDA and PSD-95 OE NMDA. Statistics not shown on graph: ${ }^{* * *} p<0.001$ at 0,5, 10 min with NMDA treatment, and after NMDA treatment followed by 10 min recovery for control vehicle versus control NMDA; *** $p<0.001$ at 10 min with NMDA treatment and ${ }^{*} p<0.05$ at 5 min with NMDA treatment for PSD-95 OE vehicle versus PSD-95 0E NMDA. No significant difference for control vehicle versus PSD-95 0E vehicle at all time points. $\boldsymbol{E}$, Number of protrusions per micrometer of dendrite. Sixty dendrites were analyzed at each time point of each group. ${ }^{*} p<0.05,{ }^{* *} p<0.01,{ }^{* * *} p<0.001$ with two-way ANOVA followed by Bonferroni multiple-comparisons test.

$3 A, B)$, and cypin protein is significantly lower in the NMDAtreated than control cultures at all time points (Fig. $3 A, C$ ). It was surprising to us that cypin protein expression decreased within such a short time after exposure to NMDA. We asked whether cypin is released from the cells as a result of treatment. To address this question, we collected and concentrated the medium with shock NMDA treatment, after 10 min of NMDA treatment, and after 10 min of NMDA treatment followed by 60 min recovery and analyzed cypin protein levels by Western blot analysis. Control neurons were exposed to vehicle. Interestingly, we found that the medium contained $\sim 25 \%$ of cypin levels found in cellular extract 


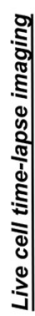

A

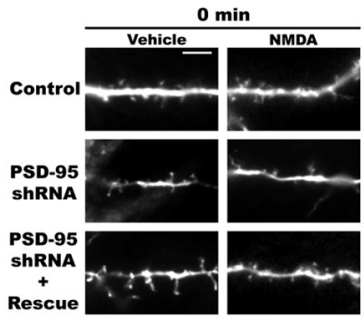

B

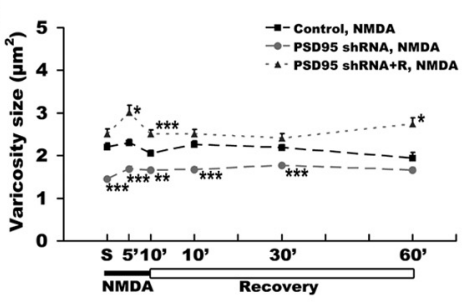

D

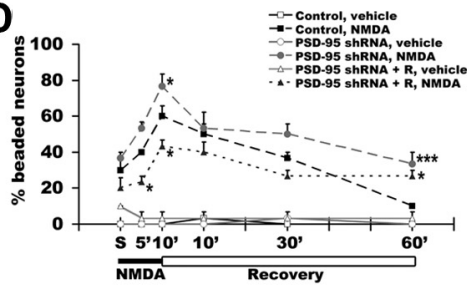

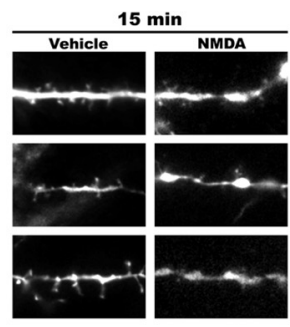

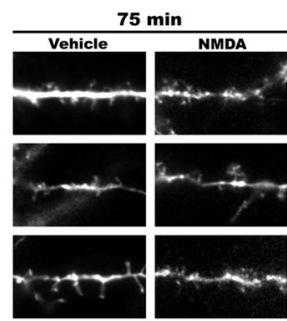

C

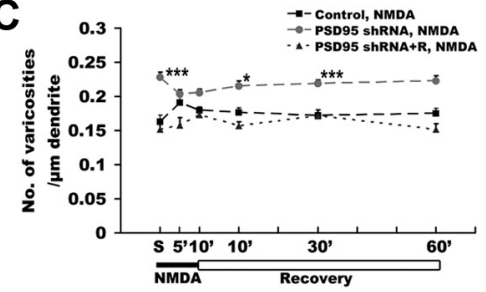

E

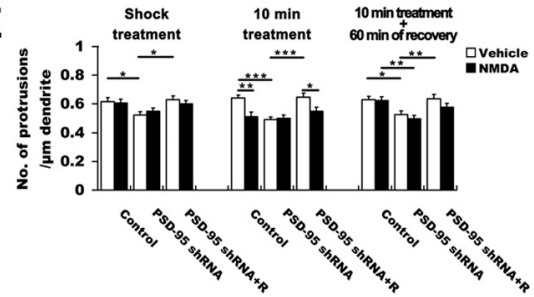

Figure 5. Effects of PSD-95 knockdown on NMDA-induced changes in dendrite morphology. Primary hippocampal neurons were transfected at DIV 14 with control shRNA (control), or PSD-95 shRNA without [PSD-95 knockdown (PSD-95 shRNA)] or with [PSD-95 knockdown plus rescue (PSD-95 shRNA + R)] CDNA encoding silent mutations in PSD-95 in the region targeted by our shRNA. At DIV 20, neurons were exposed to vehicle or $30 \mu \mathrm{m}$ NMDA for $10 \mathrm{~min}$ followed by $60 \mathrm{~min}$ in recovery medium. $A$, Representative live cell time-lapse imaging were captured at $0 \mathrm{~min}$ (before treatment), $15 \mathrm{~min}$ (end of treatment), and $75 \mathrm{~min}$ (end of recovery). Scale bar, $5 \mu \mathrm{m} . \boldsymbol{B}-\boldsymbol{E}_{i}$ Quantitative analyses of time-lapse experiment from fixed cell imaging of sister neuronal cultures from three independent experiments in $\boldsymbol{B}-\boldsymbol{D}$ and from two independent experiments in $\boldsymbol{E}$. $\boldsymbol{B}$, Varicosity size. Number of varicosities analyzed at each time point of each group is as follows: for control, $n=108$ at shock (S) with NMDA treatment, $n=157$ at 5 min with NMDA treatment, $n=197$ at 10 min with NMDA treatment, $n=206$ after NMDA treatment followed by 10 min recovery, $n=130$ after NMDA treatment followed by 30 min recovery, and $n=36$ after NMDA treatment followed by 60 min recovery; for PSD-95 shRNA, $n=172$ at $S$ with NMDA treatment, $n=224$ at 5 min with NMDA treatment, $n=324$ at 10 min with NMDA treatment, $n=177$ after NMDA treatment followed by 10 min recovery, $n=225$ after NMDA treatment followed by 30 min recovery, and $n=153$ after NMDA treatment followed by 60 min recovery; for PSD-95 shRNA $+R$, $n=52$ at $S$ with NMDA treatment, $n=65$ at 5 min with NMDA treatment, $n=154$ at 10 min with NMDA treatment, $n=129$ after NMDA treatment followed by 10 min recovery, $n=94$ after NMDA treatment followed by 30 min recovery, and $n=83$ after NMDA treatment followed by 60 min recovery. ${ }^{*} p<0.05,{ }^{* *} p<0.01,{ }^{* * *} p<0.001$ by Kruskal-Wallis test followed by Dunn's multiple-comparisons test. The plot only shows the comparison of PSD-95 shRNA NMDA or PSD-95 shRNA + R NMDA to control NMDA. Statistics not shown on graph ${ }^{* * *} p<0.001$ at all time points for PSD-95 shRNA NMDA versus PSD-95 shRNA + R NMDA. C, Number of varicosities per micrometer of dendrite. Number of beaded dendrites analyzed at each time point of each group is as follows: for control, $n=27$ at $S$ with NMDA treatment, $n=36$ at 5 min with NMDA treatment, $n=48$ at 10 min with NMDA treatment, $n=51$ after NMDA treatment followed by 10 min recovery, $n=33$ after NMDA treatment followed by 30 min recovery, and $n=9$ after NMDA treatment followed by 60 min recovery; for PSD-95 shRNA, $n=33$ at $S$ with NMDA treatment, $n=48$ at 5 min with NMDA treatment, $n=69$ at 10 min with NMDA treatment, $n=$ 36 after NMDA treatment followed by 10 min recovery, $n=45$ after NMDA treatment followed by 30 min recovery, and $n=30$ after NMDA treatment followed by 60 min recovery; for PSD-95 shRNA $+R, n=15$ at $S$ with NMDA treatment, $n=18$ at 5 min with NMDA treatment, $n=39$ at 10 min with NMDA treatment, $n=36$ after NMDA treatment followed by 10 min recovery, $n=24$ after NMDA treatment followed by 30 min recovery, and $n=24$ after NMDA treatment followed by 60 min recovery. ${ }^{*} p<0.05,{ }^{* *} p<0.01,{ }^{* * *} p<0.001$ by Kruskal-Wallis test followed by Dunn's multiple-comparisons test. The plot only shows the comparison of PSD-95 shRNA NMDA or PSD-95 shRNA+R NMDA with control NMDA. Statistics not shown on graph: not significant at $5 \mathrm{~min},{ }^{*} p<0.05$ at 10 min with NMDA treatment and after NMDA treatment followed by 10 min recovery, and ${ }^{* * *} p<0.001$ at 0 min with NMDA treatment, afterNMDA treatmentfollowed by 10 and 60 min recovery for PSD-95 shRNA NMDA versus PSD-95 shRNA + RNMDA. $\boldsymbol{D}$, Percentage beaded neurons. Thirty neurons were analyzed at each time point of each group. ${ }^{*} p<0.05,{ }^{* * *} p<0.001$ with two-way ANOVA followed by Bonferroni multiple-comparisons test. The plot only shows the comparison of PSD-95 shRNA NMDA or PSD-95 shRNA + RNMDA with control NMDA. Statistics not shown on graph: not significant after NMDA treatment followed by 10 and 60 min recovery, ${ }^{*} p<0.05$ at 0 min with NMDA treatment, and ${ }^{* * *} p<$ 0.001 at 5, 10 min with NMDA treatment, and after NMDA treatment followed by 10 min recovery for PSD-95 shRNA NMDA versus PSD-95 shRNA + RNMDA;**** $<0.001$ for control vehicle versus control NMDA in all time points except after NMDA treatment followed by 60 min recovery $(p>0.05) ; * * * 00.001$ for PSD-95 shRNA vehicle versus PSD-95 shRNA NMDA in all time points. Not significant at 0 min with NMDA treatment, ${ }^{* *} p<0.01$ at 5 min with NMDA treatment, and ${ }^{* * *} p<0.001$ at 10 min with NMDA treatment, after NMDA treatment followed by 10,30, and 60 min recovery for PSD-95 shRNA + R vehicle versus PSD-95 shRNA + R NMDA; not significant for control vehicle versus PSD-95 shRNA vehicle, control vehicle with PSD-95 shRNA + Rvehicle, and PSD-95 shRNA vehicle with PSD-95 shRNA + Rvehicle in all time points. $\boldsymbol{E}$, Number of protrusions per micrometer of dendrite. Forty dendrites were analyzed at each time point of each group. ${ }^{*} p<$ $0.05,{ }^{* *} p<0.01,{ }^{* * *} p<0.001$ with two-way ANOVA followed by Bonferroni multiple-comparisons test. under vehicle conditions; however, we detected no LDH, Tuj1, PSD-95, or GAPDH in the medium under these conditions, which would reflect general cell death (Fig. $3 D, E)$. The amount of cypin found in the medium doubled at shorter exposures to NMDA (shock, 10 min) while neither LDH nor GAPDH was released (Fig. $3 D, E$ ), implying that cypin is specifically released into the medium as a result of NMDA exposure. It is important to note that all proteins examined increased in the medium after 10 min NMDA treatment followed by $60 \mathrm{~min}$ recovery. Since there was no increase in cypin found in the media in the vehicle group (Fig. $3 D, E)$, the increases in release of all proteins most likely represent significant changes in membrane integrity in response to NMDA treatment. Thus, it appears that cypin is released from neurons at all time points and PSD-95 is released at the $60 \mathrm{~min} /$ recovery time point, suggesting that release of cypin occurs in response to NMDA treatment.

\section{PSD-95 plays a role in NMDA-induced varicosity formation}

As seen in Figures 2 and 3, the organization of microtubules is disrupted and PSD-95 protein levels are altered in response to sublethal NMDA exposure. Our previous findings show that overexpression of PSD-95 decreases dendrite branching by disrupting microtubule dynamics (Charych et al., 2006; Sweet et al., 2011a,b). Therefore, we hypothesized that alterations in PSD-95 protein expression would affect NMDAinduced varicosity formation. To test the hypothesis, PSD-95 protein was either increased (Fig. 4) or knocked down (Fig. 5) by transfecting neurons with the appropriate constructs at DIV 14. Sublethal NMDA exposure followed by live cell (Figs. $4 A, 5 A$ ) or fixed cell (Figs. $4 B-D, 5 B-D$ ) time-lapse imaging was performed at DIV 20. Live cell time-lapse imaging was used to monitor morphological changes in the same dendrites while fixed cell time-lapse imaging was used to avoid photobleaching and outof-focus imaging. Quantitative analyses of dendrite and protrusion morphology are from fixed cell time-lapse imaging. We found that sublethal NMDA treatment resulted in a significant decrease in the number of neurons with varicosities when PSD-95 was overexpressed during NMDA treatment and at the beginning of recovery (Fig. 4D). In beaded neurons, neurons overexpressing PSD-95 showed a decrease in the number of varicosities per micrometer of dendrite (Fig. 4C) and an increase in the size of varicosities (Fig. $4 B$ ) 
when compared with control neurons. In addition, the size of varicosities remained elevated when neurons overexpressing PSD-95 were returned to recovery medium for $60 \mathrm{~min}$ (Fig. $4 B$ ).

In line with these data, knockdown of PSD-95 resulted in a significant increase in the number of neurons with varicosities at the end of sublethal NMDA treatment and at $60 \mathrm{~min}$ after returning the cultures to recovery medium (Fig. 5D). An increase in the number of varicosities per micrometer of dendrite (Fig. 5C) and a decrease in the size of varicosities (Fig. $5 B$ ) were observed in neurons with PSD-95 knocked down. To demonstrate the specificity of our PSD-95 shRNA, we coexpressed a cDNA encoding silent mutations in PSD-95 in the region targeted by our shRNA. As expected, the silent mutant PSD-95 rescued the effects of PSD-95 knockdown (Fig. 5B-D). The effects of PSD-95 rescue either returned varicosity characteristics to control levels or in between the levels of control and overexpression. This is expected as we cannot control for how much knocked down PSD-95 is replaced by the silent mutant. Together, these results indicate that PSD-95 plays a role in mediating sublethal NMDA-induced varicosity formation in dendrites.

\section{Cypin plays a role in NMDA-induced varicosity formation}

Previous data from our laboratory indicate that binding of cypin to PSD-95 results in a decrease in PSD-95 clustering and delocalization (Firestein et al., 1999; Charych et al., 2006). Moreover, cypin interacts with and acts upstream of PSD-95 in dendritogenesis (Charych et al., 2006). Cypin increases dendrite branching by promoting microtubule polymerization and negatively regulating the synaptic localization of PSD-95 (Akum et al., 2004; Charych et al., 2006; Chen and Firestein, 2007). In addition, cypin protein levels are altered in response to sublethal NMDA exposure (Fig. 3). Therefore, we asked whether cypin plays a role in mediating changes in dendrite morphology after sublethal NMDA exposure. To answer this question, cypin protein was either increased (Fig. 6) or knocked down (Fig. 7) by transfecting neurons with the appropriate constructs on DIV 14. Sublethal NMDA exposure followed by live cell (Figs. $6 A, 7 A$ ) or fixed cell (Figs. $6 B-D, 7 B-D$ ) time-lapse imaging was performed on DIV 20 . We found that sublethal NMDA treatment resulted in an increase in the number of beaded neurons in neurons overexpressing cypin at the end of NMDA treatment and at the beginning of recovery (Fig. $6 D$ ). In beaded neurons, an increase in the number of varicosities per micrometer of dendrite (Fig. $6 C$ ) and a decrease in the size of varicosities (Fig. $6 B$ ) were found in neurons overexpressing cypin.

Knockdown of cypin resulted in a significant decrease in the number of beaded neurons during sublethal NMDA treatment and at the beginning of recovery (Fig. $7 D$ ). Decreased number of varicosities per micrometer of dendrite (Fig. $7 C$ ) and increased size of varicosities (Fig. $7 B$ ) were found in neurons with cypin knocked down. In addition, the size of varicosities in neurons with cypin knocked down was elevated even when cultures were returned to recovery medium for 60 min. To demonstrate the specificity of our cypin shRNA, we coexpressed a cDNA encoding a single point mutation in the 

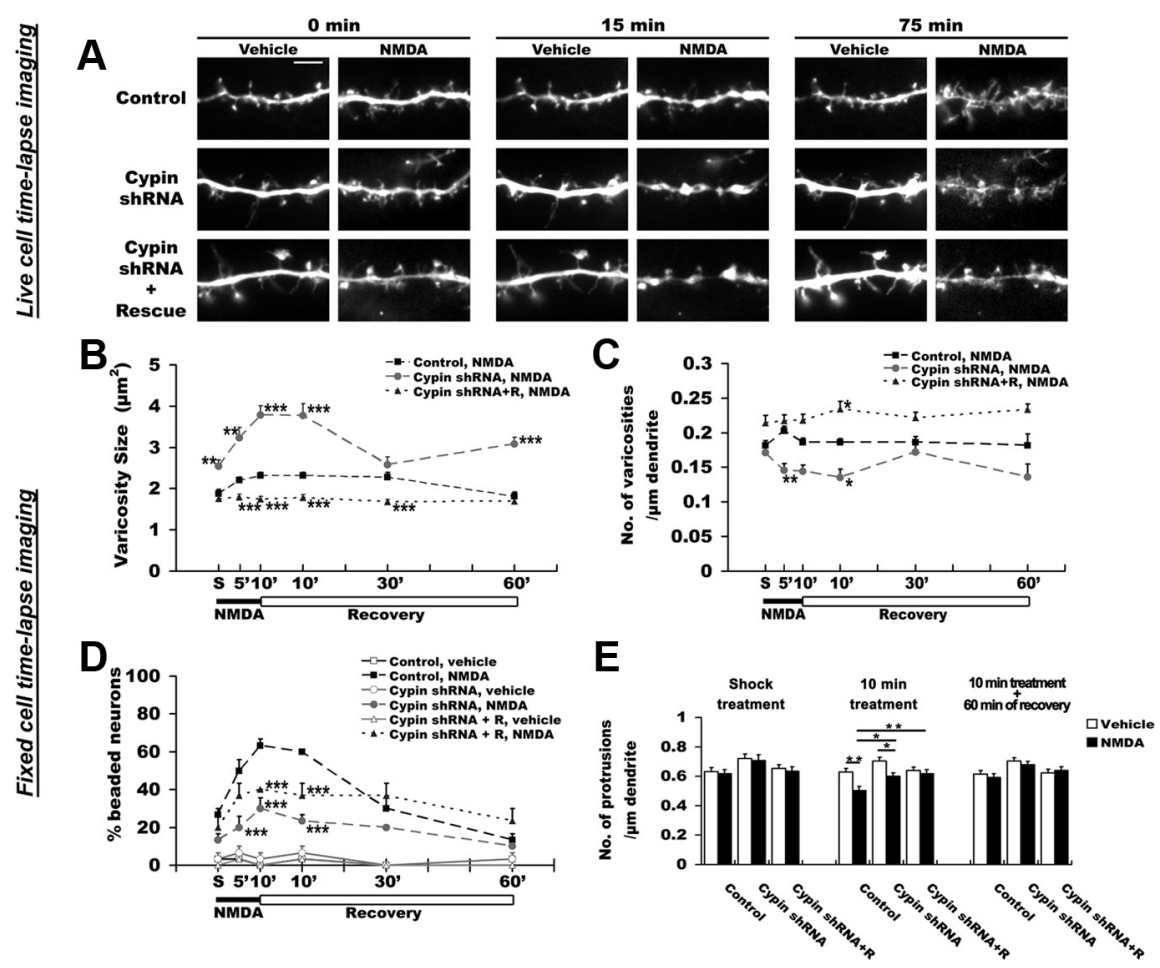

Figure 7. Effects of cypin knockdown on NMDA-induced changes in dendrite morphology. Primary hippocampal neurons were transfected at DIV 14 with control shRNA (control), or cypin shRNA without [cypin knockdown (cypin shRNA)] or with [cypin knockdown plus rescue (cypin shRNA +R)] cDNA encoding a single point mutation in the region of cypin specifically targeted by our shRNA. At DIV 20, neurons were exposed to vehicle or $30 \mu \mathrm{M}$ NMDA for $10 \mathrm{~min}$ followed by $60 \mathrm{~min}$ in recovery medium. $\boldsymbol{A}$, Representative live cell time-lapse imaging were captured at $0 \mathrm{~min}$ (before treatment), $15 \mathrm{~min}$ (end of treatment), and $75 \mathrm{~min}$ (end of recovery). Scale bar, $5 \mu \mathrm{m} . \boldsymbol{B}-\boldsymbol{E}$, Quantitative analyses of time-lapse experiments from fixed cell imaging of sister neuronal cultures from three independent experiments in $\boldsymbol{B}-\boldsymbol{D}$ and from two independent experiments in $\boldsymbol{E}$. $\boldsymbol{B}$, Varicosity size. Number of varicosities analyzed at each time point of each group is as follows: for control, $n=100$ at shock (S) with NMDA treatment, $n=209$ at 5 min with NMDA treatment, $n=243$ at 10 min with NMDA treatment, $n=230$ after NMDA treatment followed by 10 min recovery, $n=115$ after NMDA treatment followed by 30 min recovery, and $n=50$ after NMDA treatment followed by 60 min recovery; for cypin shRNA, $n=47$ at $S$ with NMDA treatment, $n=60$ at 5 min with NMDA treatment, $n=89$ at 10 min with NMDA treatment, $n=65$ after NMDA treatment followed by 10 min recovery, $n=71$ after NMDA treatment followed by 30 min recovery, and $n=28$ after NMDA treatment followed by 60 min recovery; for cypin shRNA $+R, n=88$ at $S$ with NMDA treatment, $n=164$ at 5 min with NMDA treatment, $n=179$ at 10 min with NMDA treatment, $n=177$ after NMDA treatment followed by 10 min recovery, $n=167$ after NMDA treatment followed by 30 min recovery, and $n=112$ after NMDA treatment followed by 60 min recovery. ${ }^{* *} p<0.01 ;{ }^{* * *} p<0.001$ by Kruskal-Wallis test followed by Dunn's multiple-comparisons test. The plot only shows the comparison of cypin shRNA NMDA or cypin shRNA + R NMDA with control NMDA. Statistics not shown on graph: ${ }^{* * *} p<0.001$ at all time points for cypin shRNA NMDA versus cypin shRNA + R NMDA.C, Number of varicosities per micrometer of dendrite. Number of beaded dendrites analyzed at each time point of each group is as follows: for control, $n=24$ at $S$ with NMDA treatment, $n=45$ at 5 min with NMDA treatment, $n=57$ at 10 min with NMDA treatment, $n=54$ after NMDA treatment followed by 10 min recovery, $n=27$ after NMDA treatment followed by 30 min recovery, and $n=12$ after NMDA treatment followed by 60 min recovery; for cypin shRNA, $n=12$ at $S$ with NMDA treatment, $n=18$ at 5 min with NMDA treatment, $n=27$ at 10 min with NMDA treatment, $n=21$ after NMDA treatment followed by 10 min recovery, $n=18$ after NMDA treatment followed by 30 min recovery, and $n=9$ after NMDA treatment followed by 60 min recovery; for cypin shRNA $+\mathrm{R}, n=18$ at $S$ with NMDA treatment, $n=33$ at 5 min with NMDA treatment, $n=36$ at 10 min with NMDA treatment, $n=33$ after NMDA treatment followed by 10 min recovery, $n=33$ after NMDA treatment followed by 30 min recovery, and $n=21$ after NMDA treatment followed by 60 min recovery. ${ }^{*}<<$ $0.05 ;{ }^{* *} p<0.01$ by Kruskal-Wallis test followed by Dunn's multiple-comparisons test. The plot only shows the comparison of cypin shRNA NMDA or cypin shRNA + R NMDA with control NMDA. Statistics not shown on graph: not significant at 0 min with NMDA treatment, ${ }^{*} p<0.05$ after NMDA treatment followed by 30 min recovery, ${ }^{* *} p<0.01$ after NMDA treatment followed by 60 min recovery, and ${ }^{* *} p<0.001$ at 5 min with NMDA treatment, 10 min with NMDA treatment, and after NMDA treatment followed by 10 min recovery for cypin shRNA NMDA versus cypin shRNA + R NMDA. $\boldsymbol{D}$, Percentage beaded neurons. Thirty neurons were analyzed at each time point of each group. ${ }^{* *} p<0.001$ with two-way ANOVA followed by Bonferroni multiplecomparisons test. The plot only shows the comparison of cypin shRNA NMDA or cypin shRNA + RNMDA to control NMDA. Statistics not shown on graph: not significant at 0 min with NMDA treatment, 10 min with NMDA treatment, after NMDA treatment followed by 10 and 60 min recovery, and ${ }^{*} p<0.05$ at 5 min with NMDA treatment and after NMDA treatment followed by 30 min recovery for cypin shRNA NMDA versus cypin shRNA + R NMDA; ${ }^{* * *} p<0.001$ for control vehicle versus control NMDA in all time points except after NMDA treatment followed by 60 min recovery $(p>0.05)$; not significant at 0,5 min with NMDA treatment and after NMDA treatment followed by 60 min recovery, ${ }^{*} p<0.05$ after NMDA treatment followed by 10 min recovery, ${ }^{* *} p<0.01$ after NMDA treatment followed by 30 min recovery, and ${ }^{* * *} p<0.001$ at 10 min with NMDA treatment for cypin shRNA vehicle versus cypin shRNA NMDA; ${ }^{* * *} p<0.001$ for cypin shRNA + R vehicle versus cypin shRNA + R NMDA at all time points except 0 min with NMDA treatment $\left({ }^{* *} p<0.01\right)$; not significant for control vehicle versus cypin shRNA vehicle, control vehicle versus cypin region of cypin specifically targeted by our shRNA. As expected, the silent mutant rescued the effects of knockdown (Fig. $7 B-D$ ), returning varicosity characteristics to control levels or in between the levels of control and overexpression. Thus, our data suggest that cypin regulates morphological changes in dendrites seen in response to sublethal NMDA exposure.

PSD-95 and cypin play a role in changes in dendritic protrusions in response to sublethal NMDA exposure

The regulation of PSD-95 expression and localization influences the morphological changes of dendritic protrusions, including stabilization of filopodia, maturation of spines, and density of spines (Okabe et al., 1999; El-Husseini et al., 2000; Prange and Murphy, 2001). In addition, overexpression of cypin in hippocampal neurons specifically attenuates the postsynaptic trafficking of PSD-95 (Firestein et al., 1999). Accordingly, we asked whether altering PSD-95 and cypin protein levels changes dendritic protrusion density since protrusions retracted and reemerged in response to sublethal NMDA exposure (Fig. 1 ). To address this question, we transfected neurons with appropriate constructs to modify PSD-95 and cypin protein expression on DIV 14. Sublethal NMDA exposure with live cell (Figs. 4-7A) or fixed cell (Figs. 4-7E) time-lapse imaging was performed on DIV 20. We then analyzed both spines and filopodia together as total protrusions to avoid excluding any changes in spine morphology as a result of the NMDA treatment (Hasbani et al., 2001). Overexpression of PSD-95 increased protrusion density (Fig. $4 E$ ) while knocking down PSD-95 decreased protrusion density (Fig. 5E). As expected, the silent mutant of PSD-95 rescued the effects of PSD-95 knockdown. Similarly, overexpression of cypin resulted in a significant decrease in protrusion density (Fig. $6 E$ ) while knockdown of cypin marginally increased protrusion density (Fig. 7E; $p=$ 0.056 at start of treatment and $p=0.0578$ at $60 \mathrm{~min}$ at recovery). As expected, coexpression of the silent mutation of cypin rescued the effects of knockdown.

We then assessed the effects of overexpression and knockdown of these proteins

shRNA $+R$ vehicle, and for cypin shRNA vehicle versus cypin shRNA + R vehicle at all time points. $\boldsymbol{E}$, Number of protrusions per micrometer of dendrite. Forty dendrites were analyzed at each time point of each group. ${ }^{*} p<0.05 ;{ }^{* *} p<0.01$ with two-way ANOVA followed by Bonferroni multiple-comparisons test. 
A

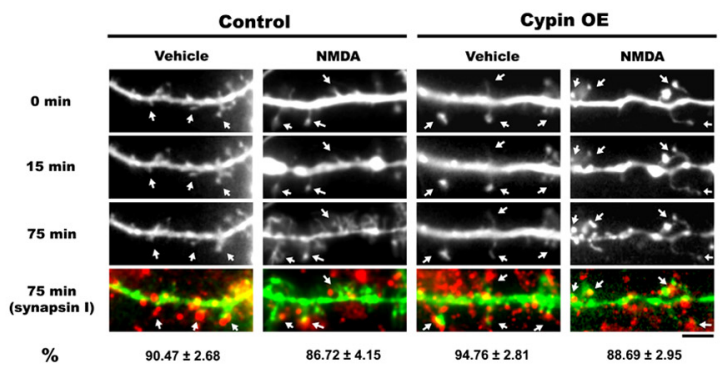

B

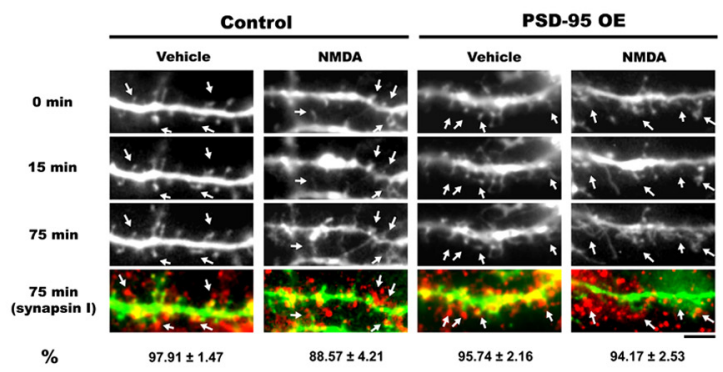

C
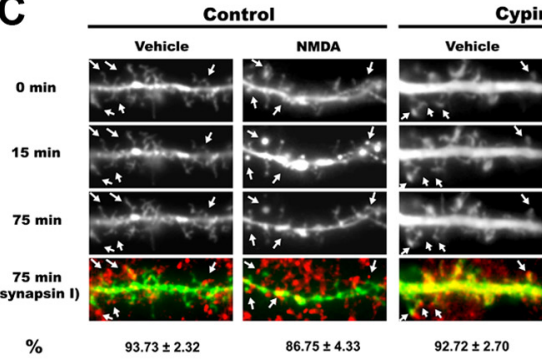

Cypin ShRNA

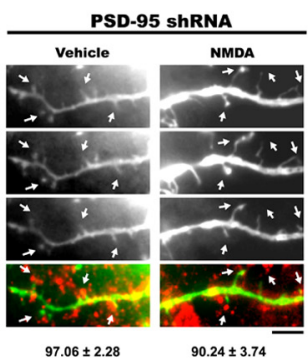

D

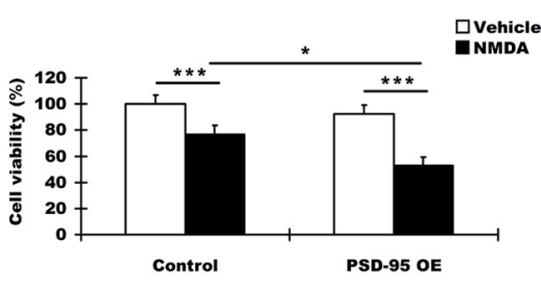

E

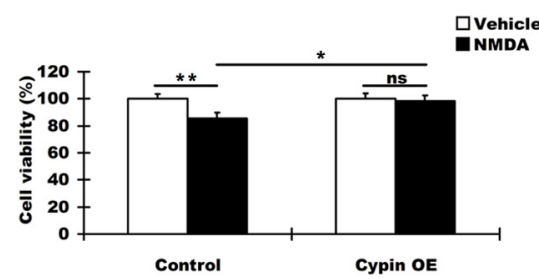

F

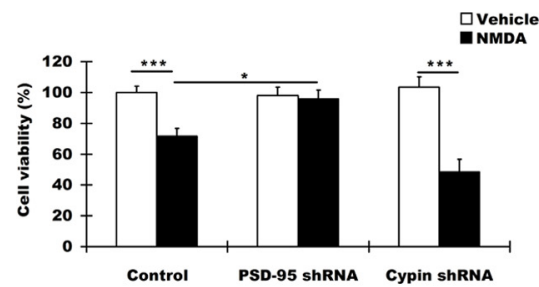

G

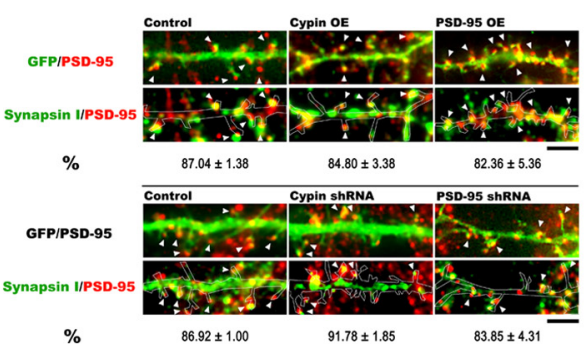

Figure 8. Synaptic connections remain during morphological changes, and cypin promotes neuroprotection. $A-C$, Primary hippocampal neurons were transfected with indicated constructs to overexpress or knockdown cypin or PSD-95 at DIV 14. At DIV 20, neurons were exposed to vehicle or $30 \mu \mathrm{m}$ NMDA for 10 min followed by 60 min in recovery medium. Representative live cell time-lapse imaging were captured at $0 \mathrm{~min}$ (before treatment), $15 \mathrm{~min}$ (end of treatment), and $75 \mathrm{~min}$ (end of recovery). At the end of recovery, neurons were fixed and stained with anti-GFP and anti-synapsin I (presynaptic marker). Arrows indicate protrusions that appose synapsin I-immunoreactive boutons and their corresponding position during live cell time-lapse imaging. Numbers below the images represent the percentage of protrusions that appose synapsin I clusters (\%). At least three live cell time-lapse images were examined. There are no significant differences between groups in each panel as determined by two-way ANOVA followed by Bonferroni multiple-comparisons test. Scale bars: $5 \mu \mathrm{m}$. D-F, At DIV 20, transfected neurons were treated with vehicle or $30 \mu \mathrm{m}$ NMDA for $10 \mathrm{~min}$ followed by $24 \mathrm{~h}$ in recovery medium. Neurons were fixed and stained with anti-GFP, anti-MAP2, and DAPI. GFP immunostaining was performed to determine the transfected cells. MAP2 and DAPI staining was performed to determine cell survival. Thirty objective views were chosen at random from three independent experiments. Overexpressing cypin and knocking down PSD-95 protects neurons from NMDA-induced excitotoxicity while knocking down cypin and overexpressing PSD-95 have opposite effects. Nonsignificant, ${ }^{*} p<0.05$, ${ }^{* *} p<0.01$, ${ }^{* * *} p<0.001$ by Kruskal-Wallis test followed by Dunn's multiple-comparisons test. G, At DIV 20, transfected neurons were fixed and stained with anti-GFP, anti-synapsin I, and anti-PSD-95. Numbers below the images represent the percentage of PSD-95 clusters that appose synapsin I immunostaining versus total number of clusters (\%). Outlines in bottom images represent dendrite as determined by GFP image. Arrows indicate PSD-95 clusters that appose to synapsin I-immunoreactive boutons and their corresponding position in protrusions. At least three live cell time-lapse images were examined. The majority of PSD-95 clusters appose synapsin I immunostaining, and this does not change when cypin or PSD-95 protein levels are altered, as determined by Kruskal-Wallis test followed by Dunn's multiple-comparisons test. Scale bars: $5 \mu \mathrm{m}$.

on changes in protrusion density in response to sublethal NMDA exposure. Overexpression of PSD-95 blocked the decrease in protrusion density seen in response to NMDA (Fig. 4E). Knockdown of PSD-95 occluded changes in protrusion density mediated by NMDA treatment (Fig. 5E). As expected, coexpression of the silent mutant of PSD-95 rescued the effects of PSD-95 knockdown. Similarly, overexpression of cypin occluded NMDA-mediated changes in protrusion density (Fig. $6 E$ ). In contrast, knockdown of cypin resulted in an attenuated decrease in protrusion density at the end of NMDA exposure (Fig. 7E). As expected, coexpression of the silent mutant of cypin rescued the effects of PSD-95 knockdown. Together, our data suggest that PSD-95 and cypin play a role in mediating changes in protrusion retraction and reemergence in response to sublethal NMDA exposure.
Presynaptic and postsynaptic elements remain in contact during dendritic changes in response to NMDA

The Goldberg laboratory has previously shown that presynaptic boutons remain associated with or near postsynaptic components during morphological changes in cortical neurons in response to NMDA treatment (Hasbani et al., 2001). Accordingly, we asked whether elimination of dendritic spines is associated with loss of synaptic contact using live cell time-lapse imaging in our in vitro model of sublethal NMDA-induced excitotoxicity, and whether altering cypin or PSD-95 protein expression influences synaptic contact during morphological change. It should be noted that regardless of PSD-95 or cypin protein levels, PSD-95 clusters are synaptic (Fig. 8G). We transfected neurons with the appropriate constructs to modify PSD-95 and cypin protein ex- 
pression on DIV 14. Sublethal NMDA exposure and live cell time-lapse imaging was performed on DIV 20. After $60 \mathrm{~min}$ in recovery, neurons were fixed and immunostained with the presynaptic marker synapsin I followed by analysis of percentage of protrusions that appose synapsin I immunostaining. As shown in Figure $8 A-C$, the majority of protrusions remained associated with presynaptic elements regardless of whether cypin or PSD-95 was overexpressed or knocked down. There was no significant difference between synaptic contacts made in the control versus overexpression and knockdown cultures.

\section{Overexpression of cypin and knockdown of PSD-95 protect neurons from NMDA-induced cytotoxicity}

A number of studies have shown that increases in the number of beaded neurons or the formation of varicosities found in neurons after glutamate exposure indicates unhealthy or dying neurons (Inoue and Okada, 2007; Meller et al., 2008; Hou et al., 2009). Others have reported the opposite effects, that varicosity production is a self-protective response against excitotoxicity and that prevention of focal swelling increases the level of cell death (Ikegaya et al., 2001). Moreover, knocking down PSD-95 protein levels modulates NMDA-induced excitotoxicity (Fan et al., 2009). Thus, we asked whether NMDA-induced neurotoxicity is affected when PSD-95 or cypin protein levels are altered. We transfected neurons with the appropriate constructs on DIV 14. At DIV 20, neurons were treated with $30 \mu \mathrm{M}$ NMDA and were returned to recovery medium for $24 \mathrm{~h}$ followed by GFP and MAP2 immunostaining and DAPI staining. MAP2 and DAPI staining was used to determine cell survival or death. Cell viability was analyzed in GFP-positive neurons with clear neuronal morphology in each condition. As shown in Figure $8 D-F$, overexpressing cypin or knocking down PSD-95 significantly protects neurons from NMDA-induced excitotoxicity, while knocking down cypin or overexpressing PSD-95 potentiates neuronal death. Furthermore, changes in cell viability are not due to aberrant subcellular localization of PSD-95 that may interfere with the NMDA receptor signaling, further influencing neuron survival rates (Sattler et al., 1999; Aarts et al., 2002; Cui et al., 2007). As shown in Figure 8G, the majority of PSD-95 clusters are synaptic in all conditions. Together, our data suggest that the promotion of smaller varicosities by increased cypin or decreased PSD-95 is neuroprotective.

\section{Discussion}

High levels of glutamate are found after traumatic brain injury (Nilsson et al., 1990; Hillered et al., 1992; Bullock et al., 1998), neurological disorders (Sloviter and Dempster, 1985; Olney et al., 1986; Rothstein, 1995), and stroke (Meldrum et al., 1985; Rothman and Olney, 1986; Lee et al., 2000). These high levels result in overstimulation of glutamate receptors, which leads to neurotoxicity. Focal swelling of dendrites and retraction of dendritic spines are early characteristics of glutamate-induced excitotoxicity (Hasbani et al., 1998, 2001; Inoue and Okada, 2007). Several in vivo and in vitro models of glutamate-induced excitotoxicity mimic varicosity formation and the loss of dendritic spines (Park et al., 1996; Faddis et al., 1997; Hasbani et al., 1998, 2001; Zhang et al., 2005; Brown et al., 2007; Durukan and Tatlisumak, 2007; Inoue and Okada, 2007; Brown and Murphy, 2008; Brown et al., 2008; Li and Murphy, 2008; Murphy et al., 2008). For example, in the Rose Bengal mouse cortical photothrombosis model, which

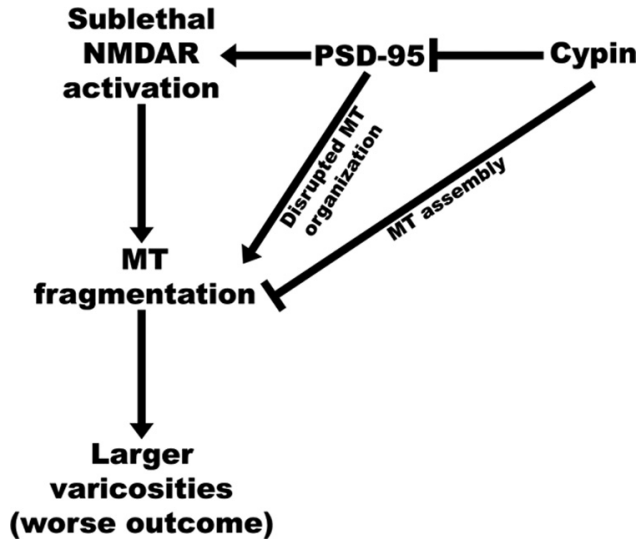

Figure 9. Possible protective mechanism in mediating sublethal NMDA-induced dendrite morphological changes. Sublethal NMDA receptor (NMDAR) activation results in microtubule (MT) fragmentation. More severe MT fragmentation produces larger varicosities, which worsens outcome after ischemia (Davies et al., 2007; Inoue and 0kada, 2007). PSD-95 disrupts microtubule organization (Charych et al., 2006; Sweet et al., 2011a,b), resulting in larger varicosities. Cypin negatively regulates synaptic PSD-95 localization and promotes microtubule polymerization (Firestein et al., 1999; Akum et al., 2004; Charych et al., 2006), producing smaller varicosities that were detected.

produces severe ischemia in vivo, spines and dendrites are rapidly damaged within 10-30 min of stroke. However, after recovery, dendritic and spine structures are mostly restored within 20-60 min (Zhang et al., 2005; Brown et al., 2007; Durukan and Tatlisumak, 2007; Brown and Murphy, 2008; Brown et al., 2008; Li and Murphy, 2008; Murphy et al., 2008). In primary mouse cortical neuronal culture, sublethal oxygen-glucose deprivation or exposure to NMDA, glutamate, or kainate causes dendritic varicosity formation within minutes of treatment. Normal dendrite and spine morphology is restored within $2 \mathrm{~h}$ after washout of the glutamate receptor activator or upon returning to normal glucose-oxygen concentrations (Park et al., 1996; Faddis et al., 1997; Hasbani et al., 1998, 2001; Inoue and Okada, 2007). Thus, dendrites and spines are disrupted within minutes after the onset of hypoxia-ischemia, while dendritic structures are restored and spines reemerge immediately at the onset of reperfusion. Much of what we know about the dendritic changes that occur in response to glutamate exposure has been gained from stroke models. This does not imply that our results are solely relevant to stroke or that we are mimicking stroke in a cell culture model, since stroke, as well as other injuries and disorders, are complicated. However, recently, a number of groups have begun to focus on understanding the recoverable dendritic morphological changes that occur during ischemia, including varicosity formation and dendritic spine retraction in surviving neurons in the penumbra. These morphological changes are thought to protect the neurons and result in behavioral and functional improvements (Kolb and Gibb, 1993; Hasbani et al., 2001; Zhang et al., 2005; Brown et al., 2007, 2008; Durukan and Tatlisumak, 2007; Brown and Murphy, 2008; Li and Murphy, 2008). Here, we established and report an in vitro model of sublethal NMDA exposure in primary hippocampal cultures that mimics changes seen in vivo. We use this model to show that PSD-95 and cypin play a role in both dendritic varicosity formation and alterations in spine and filopodia density, with opposite effects. Furthermore, we show that the formation of smaller varicosities correlates with neuroprotection. A model summarizing our results is shown in Figure 9. 
Sublethal activation of the NMDA receptor results in microtubule fragmentation, which produces larger varicosities, worsening the outcome after ischemia (Davies et al., 2007; Inoue and Okada, 2007). PSD-95 disrupts microtubule organization (Charych et al., 2006; Sweet et al., 2011a,b), therefore producing large varicosities. Cypin negatively regulates synaptic PSD-95 localization and promotes microtubule polymerization (Firestein et al., 1999; Akum et al., 2004; Charych et al., 2006), resulting in smaller varicosities.

Fragmented microtubules and loss of microtubule-associated proteins, such as MAP2, have been found in dendrites after ischemic injury (Yamamoto et al., 1986; Tomimoto and Yanagihara, 1992; Emery and Lucas, 1995; Greenwood et al., 2007). We found that microtubule dynamics change with NMDA treatment (Fig. 2 ) and that nocodazole worsened and Taxol blocked NMDAinduced microtubule breakdown. The finding using Taxol is consistent with previous findings (Jacobs and Stevens, 1986; Emery and Lucas, 1995; Park et al., 1996). Until now, very little was known about the intracellular mediators of the changes in microtubules during stroke. PSD-95 disrupts microtubule organization (Charych et al., 2006; Sweet et al., 2011a,b), and this disruption translates into decreased number of beaded neurons with increased varicosity size. Cypin promotes microtubule assembly (Akum et al., 2004; Chen and Firestein, 2007), translating into a larger number of beaded neurons with smaller varicosities. Thus, by understanding how PSD-95 and cypin affect varicosity formation, we can now identify how microtubules change during this process.

Cypin contains a domain that has high homology to collapsin response mediator protein-2 (CRMP-2), and this domain is responsible for binding tubulin heterodimers for the promotion of microtubule assembly (Firestein et al., 1999; Akum et al., 2004). Interestingly, overexpression of CRMP-2 increases axonal resistance to glutamate-induced varicosity formation (Hou et al., 2009). Like cypin, CRMP-2 promotes microtubule assembly (Fukata et al., 2002), and, together, this report and our data suggest that promotion of microtubule assembly moderates dendritic swelling. What does varicosity formation mean for the neuron? A number of studies show that a reduction of beaded neurons or number of varicosities promotes functional recovery (Inoue and Okada, 2007; Meller et al., 2008; Hou et al., 2009). Others have reported the opposite and indicate that varicosity production is a self-protective response against excitotoxicity and that prevention of focal swelling increases the level of cell death (Ikegaya et al., 2001). We have found that cypin increases varicosity production (Fig. 6), which may be neuroprotective. Similar results, including the formation of smaller varicosities, were found when PSD-95 was knocked down (Fig. 5). Smaller varicosity size has been shown to indicate a metabolically healthier hippocampus in ischemic stroke (Davies et al., 2007), and indeed, our results support this idea (Figs. 4-8). Previous findings have shown that swelling in distal dendrites occurs first, followed by swelling in proximal dendrites (Park et al., 1996; Oliva et al., 2002). The increased number of varicosities can be explained by the fact that cypin overexpression, which results in decreased PSD-95 clustering, results in increased number of distal dendrites (Firestein et al., 1999; Chen and Firestein, 2007). In line with these results, overexpression of PSD-95 and knockdown of cypin resulted in a smaller number of varicosities, which were larger in size (Figs. 4, 7). Moreover, persistent beading, as is seen with overexpression of PSD-95 and knockdown of cypin, implies an impaired regulation of dendritic volume after NMDA exposure (Inoue and Okada, 2007).
It is also interesting to note that cypin is released in response to NMDA exposure (Fig. 3). What is the significance of this finding? Release of cypin from the neuron during and after NMDA treatment may lead to cell death since intracellular cypin appears to confer neuroprotection from this treatment. However, exogenously added cypin (guanine deaminase) protects spinal motor neurons from glutamate-induced toxicity (Hedlund et al., 2010). We added exogenous cypin to our cultures and observed no protection from NMDA-induced toxicity (data not shown). This difference in the effect of cypin may be due to the distinct neuronal types or the fact that glutamate, as used in the spinal cord study, affects multiple receptor subtypes, which may contribute to injury.

What is the role of changes in dendritic spine density in response to NMDA? The collapse of spines as an early response to reduce NMDA receptor activity may serve to render neurons less vulnerable to excitotoxic stimulus (Halpain et al., 1998). In line with this theory, a decrease in the number of dendritic spines promoted by preconditioning ischemia protects against further spine loss resulting from NMDA-induced excitotoxicity (Meller et al., 2008). We have found that cypin decreases protrusion number on its own and occludes NMDA-mediated decreases in protrusion number. What mechanism might regulate this? The NMDA receptor interacts with small GTPases, and in specific RhoA, which are involved in regulation of spine number and morphology (Nakayama et al., 2000; Calabrese et al., 2005; Govek et al., 2005). We have previously reported that the activation of RhoA inhibits translation of cypin protein (Chen and Firestein, 2007) and that overexpression of cypin results in decreased PSD-95 clustering at the synapse (Firestein et al., 1999). Since it is believed that reducing the size of the postsynaptic density (i.e., reducing PSD-95), the number of synaptic NMDA receptor clusters is decreased, protecting the neurons from ischemic damage (Gisselsson et al., 2010).

We have identified a novel pathway by which neurons alter their dendritic morphology in response to sublethal concentrations of NMDA. We have shown that cypin and PSD-95, which have opposite effects on the microtubule cytoskeleton, also mediate opposite effects on the formation of NMDA-induced varicosities and dendritic protrusions. Last, we have provided evidence that alterations in cypin or PSD-95 protein levels that lead to smaller varicosities also confer neuroprotection from NMDA-induced toxicity.

\section{References}

Aarts M, Liu Y, Liu L, Besshoh S, Arundine M, Gurd JW, Wang YT, Salter MW, Tymianski M (2002) Treatment of ischemic brain damage by perturbing NMDA receptor- PSD-95 protein interactions. Science 298:846-850.

Akum BF, Chen M, Gunderson SI, Riefler GM, Scerri-Hansen MM, Firestein BL (2004) Cypin regulates dendrite patterning in hippocampal neurons by promoting microtubule assembly. Nat Neurosci 7:145-152.

Brown CE, Murphy TH (2008) Livin' on the edge: imaging dendritic spine turnover in the peri-infarct zone during ischemic stroke and recovery. Neuroscientist 14:139-146

Brown CE, Li P, Boyd JD, Delaney KR, Murphy TH (2007) Extensive turnover of dendritic spines and vascular remodeling in cortical tissues recovering from stroke. J Neurosci 27:4101-4109.

Brown CE, Wong C, Murphy TH (2008) Rapid morphologic plasticity of peri-infarct dendritic spines after focal ischemic stroke. Stroke 39:1286-1291.

Bullock R, Zauner A, Woodward JJ, Myseros J, Choi SC, Ward JD, Marmarou A, Young HF (1998) Factors affecting excitatory amino acid release following severe human head injury. J Neurosurg 89:507-518. 
Calabrese B, Halpain S (2005) Essential role for the PKC target MARCKS in maintaining dendritic spine morphology. Neuron 48:77-90.

Charych EI, Akum BF, Goldberg JS, Jörnsten RJ, Rongo C, Zheng JQ, Firestein BL (2006) Activity-independent regulation of dendrite patterning by postsynaptic density protein PSD-95. J Neurosci 26:10164-10176.

Chen H, Firestein BL (2007) RhoA regulates dendrite branching in hippocampal neurons by decreasing cypin protein levels. J Neurosci 27:8378-8386.

Cui H, Hayashi A, Sun HS, Belmares MP, Cobey C, Phan T, Schweizer J, Salter MW, Wang YT, Tasker RA, Garman D, Rabinowitz J, Lu PS, Tymianski M (2007) PDZ protein interactions underlying NMDA receptor-mediated excitotoxicity and neuroprotection by PSD-95 inhibitors. J Neurosci 27:9901-9915.

Davies ML, Kirov SA, Andrew RD (2007) Whole isolated neocortical and hippocampal preparations and their use in imaging studies. J Neurosci Methods 166:203-216.

Durukan A, Tatlisumak T (2007) Acute ischemic stroke: overview of major experimental rodent models, pathophysiology, and therapy of focal cerebral ischemia. Pharmacol Biochem Behav 87:179-197.

El-Husseini AE, Schnell E, Chetkovich DM, Nicoll RA, Bredt DS (2000) PSD-95 involvement in maturation of excitatory synapses. Science 290:1364-1368

Emery DG, Lucas JH (1995) Ultrastructural damage and neuritic beading in cold-stressed spinal neurons with comparisons to NMDA and A23187 toxicity. Brain Res 692:161-173.

Faddis BT, Hasbani MJ, Goldberg MP (1997) Calpain activation contributes to dendritic remodeling after brief excitotoxic injury in vitro. J Neurosci 17:951-959.

Fan J, Cowan CM, Zhang LY, Hayden MR, Raymond LA (2009) Interaction of postsynaptic density protein-95 with NMDA receptors influences excitotoxicity in the yeast artificial chromosome mouse model of Huntington's disease. J Neurosci 29:10928-10938.

Firestein BL, Brenman JE, Aoki C, Sanchez-Perez AM, El-Husseini AE, Bredt DS (1999) Cypin: a cytosolic regulator of PSD-95 postsynaptic targeting. Neuron 24:659-672.

Fukata Y, Itoh TJ, Kimura T, Ménager C, Nishimura T, Shiromizu T, Watanabe H, Inagaki N, Iwamatsu A, Hotani H, Kaibuchi K (2002) CRMP-2 binds to tubulin heterodimers to promote microtubule assembly. Nat Cell Biol 4:583-591.

Furukawa K, Mattson MP (1995) Taxol stabilizes $\left[\mathrm{Ca}^{2+}\right]$ i and protects hippocampal neurons against excitotoxicity. Brain Res 689:141-146.

Gisselsson LL, Matus A, Wieloch T (2005) Actin redistribution underlies the sparing effect of mild hypothermia on dendritic spine morphology after in vitro ischemia. J Cereb Blood Flow Metab 25:1346-1355.

Gisselsson L, Toresson H, Ruscher K, Wieloch T (2010) Rho kinase inhibition protects CA1 cells in organotypic hippocampal slices during in vitro ischemia. Brain Res 1316:92-100.

Govek EE, Newey SE, Van Aelst L (2005) The role of the Rho GTPases in neuronal development. Genes Dev 19:1-49.

Greenwood SM, Mizielinska SM, Frenguelli BG, Harvey J, Connolly CN (2007) Mitochondrial dysfunction and dendritic beading during neuronal toxicity. J Biol Chem 282:26235-26244.

Halpain S, Hipolito A, Saffer L (1998) Regulation of F-actin stability in dendritic spines by glutamate receptors and calcineurin. J Neurosci 18:9835-9844.

Hasbani MJ, Hyrc KL, Faddis BT, Romano C, Goldberg MP (1998) Distinct roles for sodium, chloride, and calcium in excitotoxic dendritic injury and recovery. Exp Neurol 154:241-258.

Hasbani MJ, Schlief ML, Fisher DA, Goldberg MP (2001) Dendritic spines lost during glutamate receptor activation reemerge at original sites of synaptic contact. J Neurosci 21:2393-2403.

Hedlund E, Karlsson M, Osborn T, Ludwig W, Isacson O (2010) Global gene expression profiling of somatic motor neuron populations with different vulnerability identify molecules and pathways of degeneration and protection. Brain 133:2313-2330.

Hillered L, Persson L, Carlson H, Ungerstedt U, Ronne-Engström E, Nilsson P (1992) Studies on excitatory amino acid receptor-linked brain disorders in rat and man using in vivo microdialysis. Clin Neuropharmacol 15 [Suppl 1 Pt A]:695A-696A.

Horne EA, Dell'Acqua ML (2007) Phospholipase C is required for changes in postsynaptic structure and function associated with NMDA receptordependent long-term depression. J Neurosci 27:3523-3534.
Hou ST, Jiang SX, Aylsworth A, Ferguson G, Slinn J, Hu H, Leung T, Kappler J, Kaibuchi K (2009) CaMKII phosphorylates collapsin response mediator protein 2 and modulates axonal damage during glutamate excitotoxicity. J Neurochem 111:870-881.

Hou XY, Zhang GY, Yan JZ, Chen M, Liu Y (2002) Activation of NMDA receptors and L-type voltage-gated calcium channels mediates enhanced formation of Fyn-PSD95-NR2A complex after transient brain ischemia. Brain Res 955:123-132.

Ikegaya Y, Kim JA, Baba M, Iwatsubo T, Nishiyama N, Matsuki N (2001) Rapid and reversible changes in dendrite morphology and synaptic efficacy following NMDA receptor activation: implication for a cellular defense against excitotoxicity. J Cell Sci 114:4083-4093.

Inoue H, Okada Y (2007) Roles of volume-sensitive chloride channel in excitotoxic neuronal injury. J Neurosci 27:1445-1455.

Jacobs JR, Stevens JK (1986) Experimental modification of PC12 neurite shape with the microtubule-depolymerizing drug Nocodazole: a serial electron microscopic study of neurite shape control. J Cell Biol 103:907-915.

Kobayashi MS, Takahashi Y, Nagata T, Nishida Y, Murata A, Ishikawa K, Asai S (2004) Screening for control genes in rat global cerebral ischemia using high-density oligonucleotide array. J Neurosci Res 76:512-518.

Kolb B, Gibb R (1993) Possible anatomical basis of recovery of function after neonatal frontal lesions in rats. Behav Neurosci 107:799-811.

Lee JM, Grabb MC, Zipfel GJ, Choi DW (2000) Brain tissue responses to ischemia. J Clin Invest 106:723-731.

Li P, Murphy TH (2008) Two-photon imaging during prolonged middle cerebral artery occlusion in mice reveals recovery of dendritic structure after reperfusion. J Neurosci 28:11970-11979.

Meldrum B, Evans M, Griffiths T, Simon R (1985) Ischaemic brain damage: the role of excitatory activity and of calcium entry. $\mathrm{Br} \mathrm{J}$ Anaesth 57:44-46.

Meller R, Thompson SJ, Lusardi TA, Ordonez AN, Ashley MD, Jessick V, Wang W, Torrey DJ, Henshall DC, Gafken PR, Saugstad JA, Xiong ZG, Simon RP (2008) Ubiquitin proteasome-mediated synaptic reorganization: a novel mechanism underlying rapid ischemic tolerance. J Neurosci 28:50-59.

Monnerie H, Shashidhara S, Le Roux PD (2003) Effect of excess extracellular glutamate on dendrite growth from cerebral cortical neurons at 3 days in vitro: Involvement of NMDA receptors. J Neurosci Res 74:688-700.

Murphy TH, Li P, Betts K, Liu R (2008) Two-photon imaging of stroke onset in vivo reveals that NMDA-receptor independent ischemic depolarization is the major cause of rapid reversible damage to dendrites and spines. J Neurosci 28:1756-1772.

Nakayama AY, Harms MB, Luo L (2000) Small GTPases Rac and Rho in the maintenance of dendritic spines and branches in hippocampal pyramidal neurons. J Neurosci 20:5329-5338.

Nilsson P, Hillered L, Pontén U, Ungerstedt U (1990) Changes in cortical extracellular levels of energy-related metabolites and amino acids following concussive brain injury in rats. J Cereb Blood Flow Metab 10:631-637.

Okabe S, Kim HD, Miwa A, Kuriu T, Okado H (1999) Continual remodeling of postsynaptic density and its regulation by synaptic activity. Nat Neurosci 2:804-811.

Oliva AA Jr, Lam TT, Swann JW (2002) Distally directed dendrotoxicity induced by kainic Acid in hippocampal interneurons of green fluorescent protein-expressing transgenic mice. J Neurosci 22:8052-8062.

Olney JW, Collins RC, Sloviter RS (1986) Excitotoxic mechanisms of epileptic brain damage. Adv Neurol 44:857-877.

Park JS, Bateman MC, Goldberg MP (1996) Rapid alterations in dendrite morphology during sublethal hypoxia or glutamate receptor activation. Neurobiol Dis 3:215-227.

Prange O, Murphy TH (2001) Modular transport of postsynaptic density-95 clusters and association with stable spine precursors during early development of cortical neurons. J Neurosci 21:9325-9333.

Rothman SM, Olney JW (1986) Glutamate and the pathophysiology of hypoxic-ischemic brain damage. Ann Neurol 19:105-111.

Rothstein JD (1995) Excitotoxic mechanisms in the pathogenesis of amyotrophic lateral sclerosis. Adv Neurol 68:7-20; discussion 21-27.

Sattler R, Xiong Z, Lu WY, Hafner M, MacDonald JF, Tymianski M (1999) Specific coupling of NMDA receptor activation to nitric oxide neurotoxicity by PSD-95 protein. Science 284:1845-1848. 
Sloviter RS, Dempster DW (1985) "Epileptic" brain damage is replicated qualitatively in the rat hippocampus by central injection of glutamate or aspartate but not by GABA or acetylcholine. Brain Res Bull 15:39-60.

Sweet ES, Tseng CY, Firestein BL (2011a) To branch or not to branch: how PSD-95 regulates dendrites and spines. Bioarchitecture 1:69-73.

Sweet ES, Previtera ML, Fernández JR, Charych EI, Tseng CY, Kwon M, Starovoytov V, Zheng JQ, Firestein BL (2011b) PSD-95 Alters Microtubule Dynamics via an Association With EB3. J Neurosci 31:1038-1047.

Tomimoto H, Yanagihara T (1992) Electron microscopic investigation of the cerebral cortex after cerebral ischemia and reperfusion in the gerbil. Brain Res 598:87-97.

Wahl AS, Buchthal B, Rode F, Bomholt SF, Freitag HE, Hardingham GE,
Ronn LC, Bading H (2009) Hypoxic/ischemic conditions induce expression of the putative pro-death gene Clcal via activation of extrasynaptic N-methyl-D-aspartate receptors. Neuroscience 158:344-352.

Wilson MT, Keith CH (1998) Glutamate modulation of dendrite outgrowth: alterations in the distribution of dendritic microtubules. J Neurosci Res 52:599-611.

Xia Z, Dudek H, Miranti CK, Greenberg ME (1996) Calcium influx via the NMDA receptor induces immediate early gene transcription by a MAP kinase/ERK-dependent mechanism. J Neurosci 16:5425-5436.

Yamamoto K, Morimoto K, Yanagihara T (1986) Cerebral ischemia in the gerbil: transmission electron microscopic and immunoelectron microscopic investigation. Brain Res 384:1-10. 